\title{
Emergence of flexible technology in developing advanced systems for post-stroke rehabilitation: A comprehensive review
}

Khan, Muhammad Ahmed; Saibene, Matteo; Das, Rig; Brunner, Iris; Puthusserypady, Sadasivan

Published in:

Journal of Neural Engineering

Link to article, DOI:

10.1088/1741-2552/ac36aa

Publication date:

2021

Document Version

Publisher's PDF, also known as Version of record

Link back to DTU Orbit

Citation (APA):

Khan, M. A., Saibene, M., Das, R., Brunner, I., \& Puthusserypady, S. (2021). Emergence of flexible technology in developing advanced systems for post-stroke rehabilitation: A comprehensive review. Journal of Neural Engineering, 18(6), [061003]. https://doi.org/10.1088/1741-2552/ac36aa

\section{General rights}

Copyright and moral rights for the publications made accessible in the public portal are retained by the authors and/or other copyright owners and it is a condition of accessing publications that users recognise and abide by the legal requirements associated with these rights.

- Users may download and print one copy of any publication from the public portal for the purpose of private study or research.

- You may not further distribute the material or use it for any profit-making activity or commercial gain

- You may freely distribute the URL identifying the publication in the public portal 
TOPICAL REVIEW • OPEN ACCESS

\section{Emergence of flexible technology in developing} advanced systems for post-stroke rehabilitation: a comprehensive review

To cite this article: Muhammad Ahmed Khan et al 2021 J. Neural Eng. 18061003

View the article online for updates and enhancements.
You may also like

HS-CNN: a CNN with hybrid convolution scale for EEG motor imagery classification Guanghai Dai, Jun Zhou, Jiahui Huang et al.

$\mathrm{BCl}$ for stroke rehabilitation: motor and bevond

Ravikiran Mane, Tushar Chouhan and Cuntai Guan

Classification of error-related potentials evoked during stroke rehabilitation trainin Akshay Kumar, Elena Pirogova,

Seedahmed S Mahmoud et al. 


\title{
Journal of Neural Engineering
}

CrossMark

OPEN ACCESS

RECEIVED

4 July 2021

REVISED

27 September 2021

ACCEPTED FOR PUBLICATION

4 November 2021

PUBLISHED

2 December 2021

Original content from

this work may be used

under the terms of the

Creative Commons

Attribution 4.0 licence.

Any further distribution

of this work must

maintain attribution to

the author(s) and the title

of the work, journal

citation and DOI.

\section{TOPICAL REVIEW}

\section{Emergence of flexible technology in developing advanced systems for post-stroke rehabilitation: a comprehensive review}

\author{
Muhammad Ahmed Khan ${ }^{1, *}\left(\mathbb{D}\right.$, Matteo Saibene ${ }^{1}, \operatorname{Rig} \operatorname{Das}^{1}\left(\mathbb{D}\right.$, Iris Brunner $^{2}(\mathbb{C}$ \\ and Sadasivan Puthusserypady ${ }^{1}$ \\ 1 Department of Health Technology, Technical University of Denmark, 2800 Kgs. Lyngby, Denmark \\ 2 Hammel Neurocenter, Department of Clinical Medicine, University of Aarhus, 8450 Hammel, Denmark \\ * Author to whom any correspondence should be addressed. \\ E-mail:mahkh@dtu.dk
}

Keywords: stroke, assistive and rehabilitation systems, flexible/stretchable electronics, e-textile, soft robotics, biosignals acquisition, functional electrical stimulation

\begin{abstract}
Objective. Stroke is one of the most common neural disorders, which causes physical disabilities and motor impairments among its survivors. Several technologies have been developed for providing stroke rehabilitation and to assist the survivors in performing their daily life activities. Currently, the use of flexible technology (FT) for stroke rehabilitation systems is on a rise that allows the development of more compact and lightweight wearable systems, which stroke survivors can easily use for long-term activities. Approach. For stroke applications, FT mainly includes the 'flexible/stretchable electronics', 'e-textile (electronic textile)' and 'soft robotics'. Thus, a thorough literature review has been performed to report the practical implementation of FT for post-stroke application. Main results. In this review, the highlights of the advancement of FT in stroke rehabilitation systems are dealt with. Such systems mainly involve the 'biosignal acquisition unit', 'rehabilitation devices' and 'assistive systems'. In terms of biosignals acquisition, electroencephalography and electromyography are comprehensively described. For rehabilitation/assistive systems, the application of functional electrical stimulation and robotics units (exoskeleton, orthosis, etc) have been explained. Significance. This is the first review article that compiles the different studies regarding FT based post-stroke systems. Furthermore, the technological advantages, limitations, and possible future implications are also discussed to help improve and advance the flexible systems for the betterment of the stroke community.
\end{abstract}

\section{Introduction}

Stroke is a neurological disorder in which the brain is unable to receive an adequate amount of oxygen due to obstruction in blood flow to the brain cells. It is a life-changing event that can affect the subject's cognitive and emotional state as much as their physical functions. Studies show that individuals recovering from a stroke often experience helplessness, frustration, and social isolation, which is linked to increased depression and decreased ability to manage their daily activities $[1,2]$. According to a study conducted in 2015, there are about 25.7 million stroke survivors worldwide [3]. One recent study indicates that there are approximately 116.4 million DALYs (disabilityadjusted life-years) and 5.5 million deaths due to stroke [4].
Mainly there are five post-stroke phases that comprise hyper-acute $(0-24 \mathrm{~h})$, acute (1-7 days), early subacute (7 days to 3 months), late subacute (3-6 months), and chronic (>6 months) $[5,6]$. Among the stroke survivors, around 50\% suffer from upper limb paresis, i.e. weakness or inability to move the upper limb [7]. Thus, the primary aim of poststroke care is to assist the patients in their everyday life activities and rehabilitate them for effective recovery of lost functions. This allows them to regain their independence and reintegrate into the social community. Currently, the most common stroke rehabilitation methods for restoring motor functions are occupational and physical therapies [8]. In these approaches, task-specific and repetitive training is performed to induce motor recovery based on motor learning and neuroplasticity mechanisms. 
Motor rehabilitation is supposed to support the brain in reorganizing its neural networks and relearning the skills that were lost due to stroke conditions $[9,10]$.

With the advancement in science and technology, new stroke rehabilitation methods have been introduced, which include the use of functional electrical stimulation (FES) and robotics assistance system [11]. FES is used as a non-invasive rehabilitation tool to restore the motor skills of stroke survivors by stimulating the targeted nerves via applying electrical impulses through the skin surface, thus, inducing movements in paretic muscles [12-14]. This method was first implemented on hemiplegia patients by Moe et al [15], which was later improved by Kralj et al [15] to treat subjects with neural disorders. Several studies confirm the efficacy of FES in recovering different muscle movements, for instance, restoring hand grasp [16-18], walking [19, 20], arm reaching $[21,22]$, standing $[23,24]$, and upper-limb rehabilitation [25-27]. On the other hand, the main objective of robotics-based rehabilitation systems in stroke therapy is to provide assistance in restoring impaired limb movements. These systems mainly include orthoses, exoskeletons, and other robotics units that allow the rehabilitation of upper and lower limbs, depending on the stroke severity [28-39]. An additional advantage of robotics systems includes the ability to measure the dynamic and kinematic parameters of the subject's motion during the therapy. This allows monitoring the subject's performance while performing the rehabilitation exercises by estimating their speed, range of motion, task execution accuracy, etc [40].

The ultimate objective of rehabilitation therapies is to restore the brain connections for motor recovery and thereby function. Therefore, along with the therapist's assistance, the subject's active participation can improve the outcomes. In this regard, the use of motor imagery (MI) paradigm of the brain-computer interface (BCI) system seems to be an innovative approach to neurorehabilitation [41-44]. MI training consists of the representation of imaginary movements of limbs without physically performing it. This ability of the brain to imagine a movement is used for restoring motor skills. MI activates some of the neural circuits that are also involved in the real movements, and thus, could induce functional redistribution of neuronal circuits $[45,46]$. An MI-BCI is a computer-based system that records the electroencephalography (EEG) signals and translates the user's intention to perform the specific task based on MI events, for example, activating the muscle stimulator or controlling a robotic rehabilitation unit. Such MIBCI systems have widely been used in stroke rehabilitation for motor and functional recovery [47-66]. Apart from MI [67], steady-state visual evoked potential (SSVEP), another BCI paradigm, has also been used for controlling assistive devices by the use of subject's EEG activities. SSVEP is produced in response to external visual stimulus and can be generated by alternating graphical patterns, flashing lights, and flickering images. Usually, their frequencies lie in the range of low $(1-3.5 \mathrm{~Hz})$ to high $(75-100 \mathrm{~Hz})$ frequency bands, and based on the frequency range, the SSVEP-BCI system executes a required action [68]. Such systems have been largely used for wheelchair applications in which the different directions of the wheelchair are maneuvered by a set of pre-defined frequency values [69-72].

In addition to EEG, the use of surface electromyography (EMG) has also been proven as an efficient approach to control rehabilitation and assistive devices. The use of EMG as feedback allows to analyze the real-time muscle activity and provides information regarding the amount of rehabilitation required [39]. For instance, in the case of FES device, the EMG module will record the muscle potential and provide feedback to the FES block for adjusting the electrical stimulation according to the requirement [73-75]. Also, EMG is used for controlling assistive devices, which comprises of controlling electric wheelchairs $[76,77]$ and robotic orthoses/exoskeletons [78-80].

The above-mentioned biosignals acquisition and rehabilitation approaches are delivering promising results; however, the current systems/devices are bulky, rigid, and need special expertise to operate them. Hence, there is a need to improve them by transforming these conventional systems into 'Smart Systems' that would be wearable, flexible, compact, lightweight, portable, and user-friendly (depending on their mode of application). Recent advances in flexible technology (FT) have offered a variety of innovative solutions to the given challenges. In neurorehabilitation applications, the term 'Flexible Technology' mainly includes the development of: (a) Flexible/stretchable electronics based bio-sensing systems/electrodes, (b) e-textile (electronic textile) based systems, and (c) Soft robotics-based flexible prototype, e.g. flexible exoskeleton/orthosis. Flexible electronics (FE) is an advanced technology that enables the fabrication and incorporation of sensors and electronic circuits on flexible, bendable, stretchable, and twistable substrates [81]. The concept of FE was introduced in the 1960s when Crabb and Treble developed the first flexible solar cells [82]. From 1990 to 2000, flexible transistors and transducers have been designed primarily by using flexible organic thin films [83-85]. Later, as the field of FE advanced, its range of biomedical applications increased, including artificial electronic skin (e-skin) [86-89], vital sign monitoring [90-92], and neural interfaces [93]. Another FT is the 'e-textile' application in which the electronics is embedded into the stretchable garments/textile to perform actuating and sensing functions $[94,95]$. In healthcare, e-textile advantages are significant and have widely been adopted in monitoring physiological parameters [96-98], biosignals acquisition [99, 100], gait and postural assessment 
[101, 102], and prosthesis control [103]. Additionally, soft robotics is in high demand nowadays due to newly added features, including the high degree of freedom (DOF) and range of motion along with attained flexibility and portability, which has never been achieved with rigid-link robotics [104]. Therefore, soft robots have contributed to the various platforms; for instance, used in performing manipulation tasks (e.g. grasping) [105, 106], mobility assistance (walking) [107], and other medical applications [108-110]. The application of FT in stroke rehabilitation and assistive systems will be described comprehensively in later sections of this manuscript.

To date, numerous review articles have been published related to the healthcare applications of FT, which comprises flexible textile electrodes for biosignals monitoring [99], wearable electronics and smart textiles [111], e-textiles in neurorehabilitation [112], wearable sensors and systems with application in rehabilitation [113], and FE for soft robotics $[114,115]$. However, none of them focuses on providing a detailed overview of different flexible methodologies for developing flexible stroke rehabilitation and assistive systems. Hence, this review article has compiled the implementation of several FTs for stroke application (FE, e-textile and soft robotics) and has comprehensively described them in terms of the development of biosignal acquisition unit and rehabilitation/assistive systems. Moreover, current limitations and future research directions are also discussed for possible improvements of flexible stroke rehabilitation systems.

\section{Searching criteria}

Before the systematic search, inclusion criteria (IC) and exclusion criteria were defined. Only papers that met all the IC listed below were selected:

IC1: The article must be written in English.

IC2: The publication date should be on or after the year 2010.

IC3: The study should be based on FT in terms of flexible electronics OR e-textiles OR soft robotics.

IC4: The study must report information about any of the following: signal acquisition approaches used for stroke systems (EEG and EMG) OR stroke rehabilitation systems (either conventional or BCI based) OR assistive systems (exoskeleton, orthosis, or other robotic units).

IC5: Among the soft robotics systems, only those studies are included that have validated their rehabilitation effect on stroke users.
EC1: The papers that involve FT but do not address its application for stroke systems.

EC2: The papers that contain the development of stroke systems without the use of FT.

To perform the systematic review, we searched for articles in PubMed, ScienceDirect, IEEE, and Scopus databases using the keywords: FE, stroke rehabilitation, BCI, brain computer interface, brain-machine interface, brain machine interface, neural-machine interface, neural machine interface, biosignal acquisition systems, e-textile, soft robotics, neurorehabilitation devices, FES, robotics systems, and assistive stroke systems. Figure 1 illustrates the overall screening process for inclusion and exclusion of the research articles. Initially, 1565 papers were found, and among them, 237 duplicates were removed (level 1). According to the IC, the remaining 1328 papers were assessed and based on their titles and abstract, 645 articles were excluded (level 2). This resulted in a total of 657 manuscripts for full-text screening, out of which only 26 research articles fulfilled the IC and are included in this review article (level 3).

\section{FT in post-stroke systems}

Post-stroke systems consist mainly of two building blocks: (a) biosignal acquisition systems to collect physiological signals like EEG and EMG, and (b) assistive and rehabilitation devices that are used to perform/assist in rehabilitation exercises (for instance, electrical stimulation devices and robotics exoskeleton). The use of bioelectronics, sensing technology, bioinstrumentation, telecommunication, and signal analysis techniques have played a vital role in developing the aforementioned systems. In the past, the system/device compactness and its transformation into wearable form was always a big question, restricting the adoption of such systems for long-term neural applications. However, the recent advancement in FT has allowed the development of flexible, stretchable, and compact systems that entail the features of signal acquisition, microcontroller operations, sensing capability, and wireless transmission [113]. The overall schematic of FT applications in stroke rehabilitation systems has been shown in figure 2 .

\subsection{Biosignal acquisition systems and electrodes} 3.1.1. Brain signals acquisition systems/electrodes Based on the process of recording brain activities, the brain acquisition techniques are of three types, i.e. invasive, semi-invasive, and non-invasive [116]. The invasive method is also called 'Intracortical Acquisition Scheme' in which the electrodes are implanted 


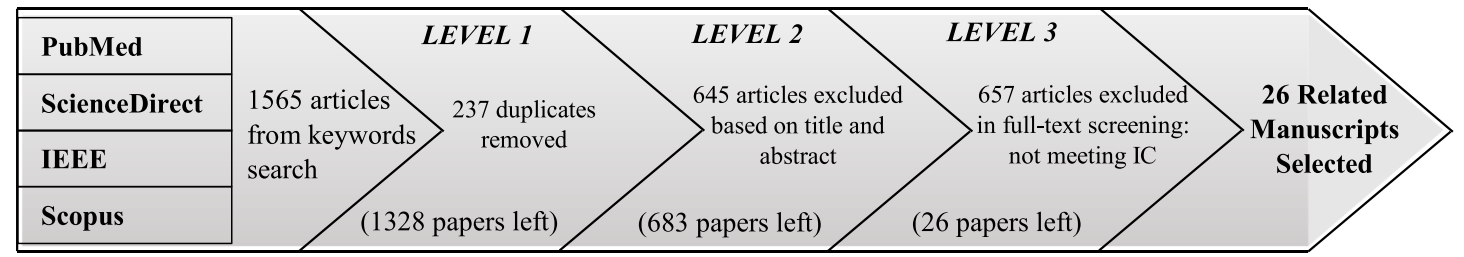

Figure 1. Literature search process and results.

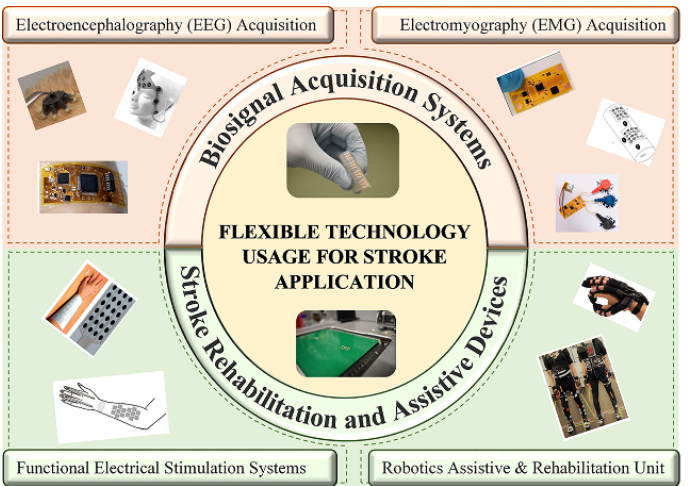

Figure 2. Overview of FT application for stroke rehabilitation systems.

into the brain's cortex to record the action potential generated by neurons firing [117]. Secondly, semi-invasive techniques are mostly used to record signals from the brain's cortical surface using electrocorticogram (ECoG) $[118,119]$. Lastly, the noninvasive technique, which does not require electrode implantation and records the signals from the brain scalp via EEG. EEG possesses high temporal resolution; however, it depicts a much lower spatial resolution and is more sensitive to external noises as compared to invasive and semi-invasive methods [120]. Non-invasive BCI systems are preferred for acquiring brain signals due to their comfort, feasibility, safety, portability, and low cost. Additionally, according to studies $[121,122]$, subjects prefer noninvasive systems for their medical diagnosis and treatment despite having low-quality signals. Therefore, the non-invasive EEG systems are the most commonly used in BCI-assisted stroke rehabilitation systems that are based on either gel [123-125] or dry electrodes [126, 127]. However, the current EEG systems [128-131] are heavy, bulky, and contain rigid hardware components, hence, not suitable for longterm mobile EEG monitoring on a daily basis. Thus, to fill this gap and to make EEG recording comfortable and feasible for day-to-day use, FE has stepped into the field of developing 'Flexible EEG Systems/ Electrodes'.

In recent research, Mahmood et al have developed a flexible, portable, and wireless EEG acquisition device (termed as 'SKINTRONICS') for BCI-assisted
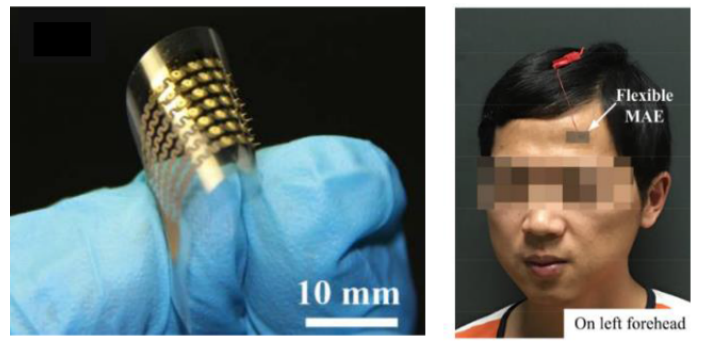

Figure 3. Flexible MAE. Reproduced from [136]. CC BY 4.0.

neurorehabilitation system [132]. The overall system includes an FE-based circuit, three flexible elastomeric electrodes for the scalp, and a skin electrode. It presents a remarkable reduction of electromagnetic and noise interference compared to standard EEG systems [131, 133-135]. The device works on the SSVEP-BCI paradigm and has been tested on six human subjects for real-time controlling of a wireless wheelchair, slide changing of presentation software, and wireless mini-vehicle. The obtained results show higher control accuracy of $94.01 \pm 3.6 \%$ and $96.24 \pm 3.4 \%$ at intervals of $0.512 \mathrm{~s}$ and $1.024 \mathrm{~s}$, respectively. In another research, a flexible microneedle array electrode (MAE) has been developed by Ren et al [136], which is ideal for biosignal monitoring, including the wearable EEG measurement. For electrode fabrication, the flexible polyethylene terephthalate substrate has been used. On the substrate, the conductive patterns and microneedle array are deposited by laser-direct writing and magneto-rheological drawing lithography techniques, respectively (figure 3). The MAE are tested for eyes blink, close and open features and their performance is compared with the standard $\mathrm{Ag} / \mathrm{AgCl}$ electrode and flexible dry electrode (FDE). The result shows that the EEG monitoring ability of MAE is similar to that of $\mathrm{Ag} / \mathrm{AgCl}$ electrodes, which proves the feasibility and possible usage of flexible MAE for biosignal measurement. Also, flexible dry EEG electrodes have been developed by Grozea et al [137] that are made of flexible metal coated polymer bristles (figures $4(\mathrm{a})-(\mathrm{c})$ ). The experimental results show that the FDEs are able to record alpha rhythms, P300 event-related potentials, MI BCI paradigms and auditory evoked potential. Moreover, 


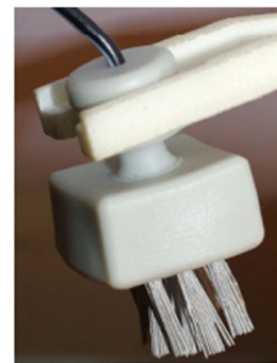

(a) Prototype

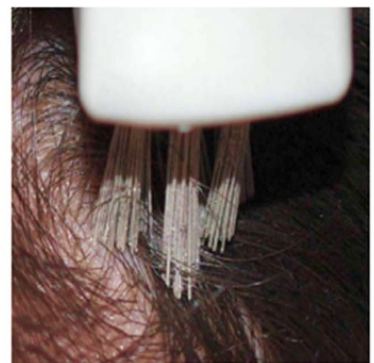

(b) Through hair



(c) Flexing

Figure 4. Silver-coated polymer bristles prototype: (a) prototype (b) through hair (c) flexing. Reproduced from [137]. ( $)$ IOP Publishing Ltd. All rights reserved.

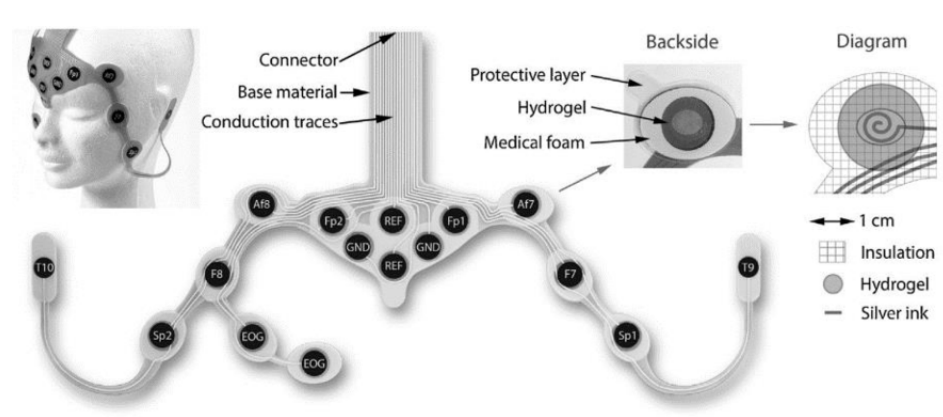

Figure 5. BrainStatus consisting of ten electroencephalography (EEG) electrodes, two electrooculography (EOG) electrodes, two ground electrodes and two reference electrodes. Reprinted from [138], Copyright (2014), with permission from Elsevier.

it is also found that the quality of EEG signals acquired from flexible electrodes are closer to the signals that are recorded using standard gel-based electrodes (within the range of $7-44 \mathrm{~Hz}$ ). Lepola et al [138] developed a flexible screen-printed EEG electrode set called 'BrainStatus' (figure 5), consisting of 16 hydrogel-coated electrodes (ten EEG recording electrodes, two electrooculography electrodes, two ground electrodes, and two reference electrodes). BrainStatus has been tested on two clinical patients, and the quality of the acquired EEG signal was excellent and comparable to the conventional EEG electrodes. Hence, this system can be feasible and able to provide effective solution for long-term EEG monitoring of subjects with stroke or other neural disorders.

Apart from conventional EEG recording systems in which the electrodes are placed on the scalp, Norton et al introduced a new flexible EEG system. The system is capable of providing long-term ( $>14$ days) EEG recordings with the electrodes positioned at the auricle (outer ear surface) and the adjacent regions (mastoid area) [139] (figure 6). This dry electrode system consisted of a collection of gold electrodes with $300 \mathrm{~nm}$ thickness and coated with a sprayon-bandage material that ensures reliable recordings during normal daily activities. Finally, the device has been tested on a group of volunteers using an SSVEPBCI text-speller and achieved an average accuracy of $93 \%$ with a spelling rate of $2.3-2.5$ letters per minute,



two to three times slower than a conventional cap EEG system on the hairy scalp.

Moreover, nowadays, the application of e-textiles technology in a biopotential acquisition is growing gradually, which allows the development of flexible and stretchable textile-based EEG monitoring electrodes [99]. However, the main limitation of applying textile electrodes in EEG recording is hair on the scalp. Therefore, e-textile electrodes are placed 
on non-hairy regions, for instance, on the forehead and behind the ears [140]. Matiko et al [141] have developed and tested a self-powered EEG headband containing integrated flexible solar panels and screen-printed conductive electrodes. The device was tested on 12 subjects, and different emotion responses were identified via EEG classification. The system performance was compared with the commercially available passive electrodes, and the obtained outcome showed a correlation result of $70.88 \%$. Recently, La et al [142] have also developed screen-printed e-textile patches, which have been tested for EEG assessment against eye-opening and closing activities, with an electrode placed behind the ear in the mastoid region. The results were quite promising and showed that the e-textile electrode contained smaller motion artifacts than commercial rigid sensors.

\subsubsection{EMG acquisition systems/electrodes}

There are two main types of EMG measurements; intramuscular EMG and surface EMG (sEMG) [143]. Intramuscular EMG is an invasive method that is used to study the deep muscles [144, 145]. It is a time-consuming process and requires special clinical expertise to insert the electrodes deep into the muscles; hence, rarely been used in practical applications [146]. On the other hand, sEMG is a non-invasive technique that acquires EMG signals from large surface areas and has widely been used for recording the electrical potential of superficial muscles [147]. sEMG has a low-signal resolution, possesses a relatively narrow frequency band $(20-500 \mathrm{~Hz})$, and is highly susceptible to movement artifacts as compared to invasive EMG [148-150]. However, the sEMG signal quality can be improved by selecting the appropriate electrode location [147], and optimum electrode size [151]. Despite having inherent limitations, sEMG is practically preferred because of its non-invasiveness [152].

As the sEMG based research for neurorehabilitation applications advances, the need of flexible and stretchable EMG electrodes arises to provide novel and advance solutions for monitoring muscle activities. The flexible EMG electrodes are more feasible than conventional electrodes, as they can be easily placed on curved body surfaces and are also comfortable for long-term myosignal recordings. Additionally, they can be embedded into wearable devices of different shapes and can minimize the overall compactness of the system [153]. In [154], flexible sEMG electrodes are used to control FES activation, which plays a vital role in delivering stroke rehabilitation. The system is tested on eight healthy subjects by positioning the flexible EMG electrodes on extensor carpi radialis, extensor digitorum communis, and extensor carpi ulnaris muscles of the forearm (figure 7). The FES is adjusted based on the muscle activities and provides sufficient stimulation for wrist extension,



Figure 7. Multi-contact set-up visualized for the left arm: Electrode array for FES with a fixed configuration of two blocks, i.e. a proximal and a distal (close to the hand) block with 15 and 12 contacts respectively, and three flexible electrodes for EMG recording. Reprinted by permission from Springer Nature Customer Service Centre GmbH: Springer Nature, Journal of Neuroengineering and rehabilitation [154]

Multi-contact functional electrical stimulation for hand opening: electrophysiologically driven identification of the optimal stimulation site, De Marchis et al, (c) 2016.

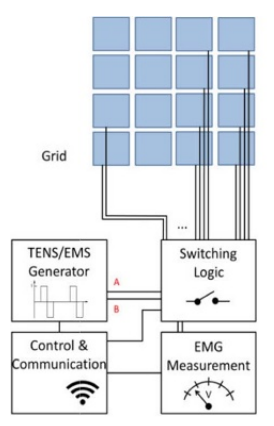

Figure 8. Components of the planned electrode grid. The EMS generator produces the stimulation signals, which are directed to different electrode configurations over the switching logic. A control and communication unit synchronizes the components. It controls the stimulation parameters and collects the measurements from the EMG unit. Reproduced with permission from [155].

hand opening, and ulnar deviation. In another related research [155], on-skin technology has been used to develop a flexible, stretchable, and power efficient sEMG electrode grid for controlling FES stimulation (figure 8). Xu et al [156] developed a flexible skin-mounted sensing platform that can monitor sEMG via sensing, ground, and reference electrodes (figure 9(a)). The designed sEMG sensing platform is fabricated as a flexible electronic skin tattoo containing thin gold and polyimide layers. The electronic tattoo is attached to the biceps and triceps surface, and depending on the generated muscle signals, the extension and flexion of the robotic arm's elbow joint are controlled (figures 9(b) and (c)). Thus, such a flexible sEMG controlled robotic control system can be implemented to develop robotic assistive devices for stroke rehabilitation. Fall et al [157] developed a smart textile based sEMG electrodes, made of metal polymer-glass hollow-core fiber. The electrodes testing are performed by recording the muscle activities of biceps and forearm flexor muscles. 

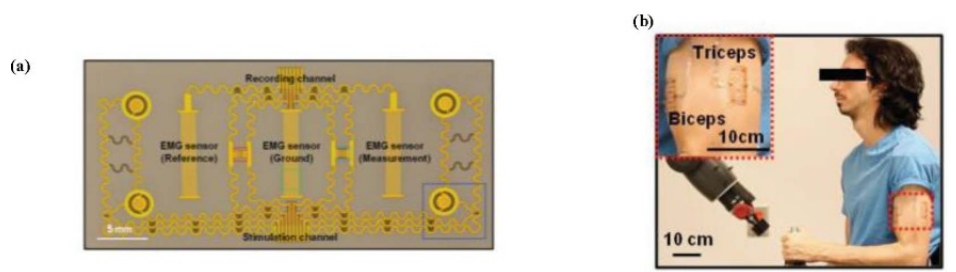

Figure 9. (a) Simple, multifunctional device with skin-like physical characteristics and capabilities in both sensing and stimulation (b) devices on the bicep and triceps (inset) during control of the angle of the elbow of a robot arm [156]. John Wiley \& Sons. (c) 2015 WILEY-VCH Verlag GmbH \& Co. KGaA, Weinheim.
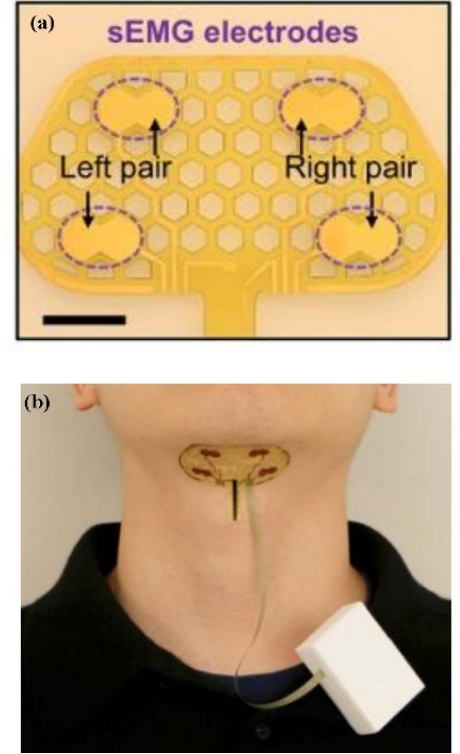

Figure 10. Basic layouts and configurations of the sensor patch system. (a) Enlarged photo of the sEMG recording electrodes. Scale bar, $1 \mathrm{~cm}$. (b) Schematic illustration of the sensor patch connected with a portable unit clipped on the wearer's clothing for remote data transmission and powering. From [158]. Reprinted with permission from AAAS.

The obtained results confirm that the smart sEMG electrodes possess the performance similar to commercial gold-plated electrodes. Hence, during stroke rehabilitation, such sEMG units can be used as a biofeedback. It can provide real-time data of muscular activities and command the rehabilitative robots to provide assistance based on the muscle requirement. Moreover, in [158], a skin-mountable flexible sensor patch has been developed for the patients suffering from dysphagia (difficulty swallowing disorder), which is caused by different neural disorders, including the stroke [159]. The patch is designed for the submental area (under the chin) to provide the remote monitoring of muscle activity (sEMG) during the swallowing tasks (figure 10). The monitoring of swallowing events during the post-stroke rehabilitation of dysphagia is very important as it provides information regarding the level of muscular improvement and allows to adjust the rehabilitation exercises accordingly. Preliminary testing on a patient with dysphagia, and on a healthy control validates the effectiveness and feasibility of this system.

\subsubsection{Overview of biosignals acquisition systems}

Table 1 shows an overview of different studies in which FT has been used to develop electrodes and systems for measuring EEG and EMG signals.

\subsection{Stroke rehabilitation and assistive devices 3.2.1. FES based rehabilitation}

It has been shown that the upper limb stroke rehabilitation performed via the FES technique results in better performance as compared to the physical therapy alone [171-174]. For an effective implementation of FES in stroke rehabilitation, the vital parameters of stimulation (onset therapy time [172, 175] and dosage $[176,177]$ ) should be chosen with great caution. Apart from having significant advantages, the current FES devices possess the limitation of providing 'Selective Stimulation'. For instance, when focusing on the recovery of a particular hand function, it is necessary to position the electrodes precisely over the muscle motor point to produce specific muscular contraction [178]. The motor point is an 'optimized electrode area' where the required stimulation effect is attained with minimal electrical stimulation. As current FES devices normally use a pair of large gel electrodes, several current paths are produced under the applied electrodes, stimulating different muscles. This causes the compromising of selective activation of targeted muscles and also induces muscle fatigue [179]. Hence, to overcome this shortcoming and provide selective stimulation, the flexible multiple electrodes array has been developed to easily be placed on curvy surfaces and cover multiple targeted areas on a single location [154, 155, 180-183]. It allows the selective activation of individual electrodes to deliver the selective stimulation to targeted muscles. Additionally, studies showed the spatial distribution of stimulation across multiple electrodes also delays the onset of muscle fatigue [184-186].

Yang et al [180] developed an e-textile based flexible 24-electrode array called 'e-sleeve' for FES rehabilitation device (figure 11). The e-sleeve has been fabricated via screen printing technique and can cover multiple muscle groups, eliminating the need for precise positioning of electrodes on the targeted 


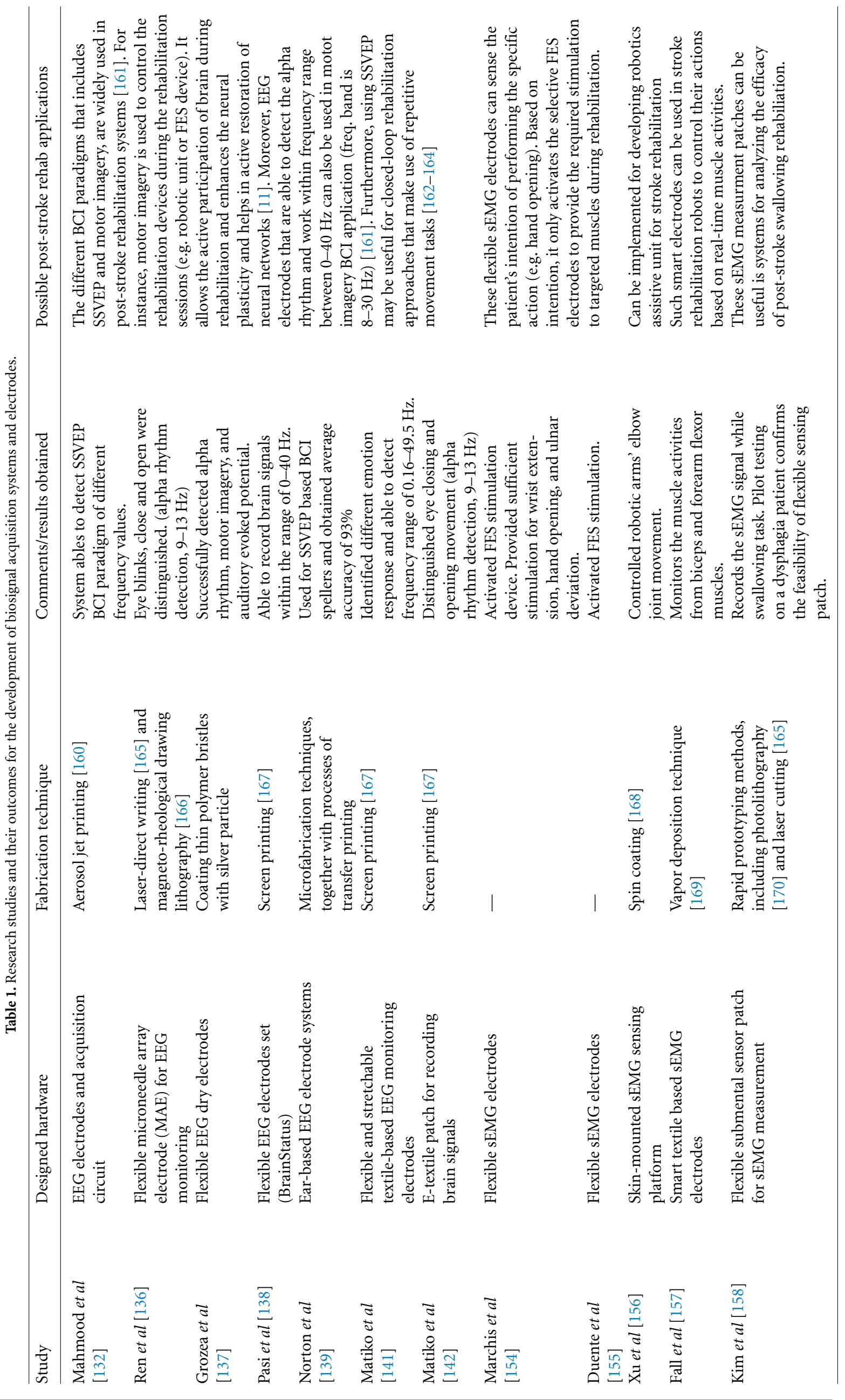




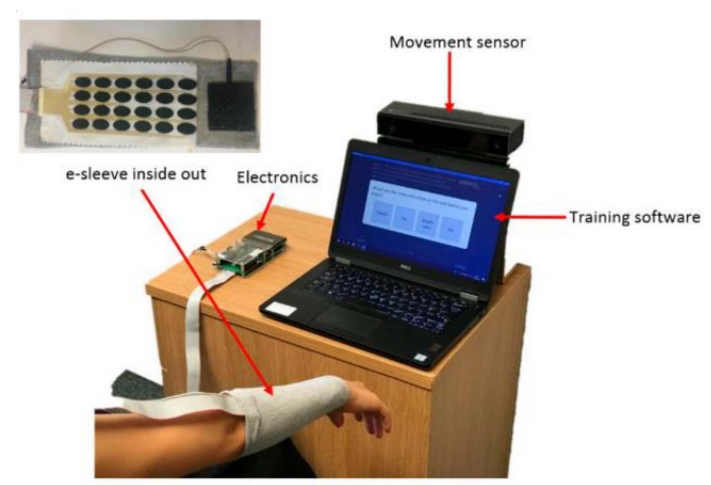

Figure 11. E-sleeve and FES training system. Reproduced from [180]. CC BY 4.0.

muscle. The developed algorithm activates the optimized combination of electrodes required to perform specific movements. The e-sleeve performance was tested on eight stroke survivors with upper limb disability by performing 'hand opening and pointing' actions. Result shows that the system selected the right combination of electrode array to achieve the targeted motion. In [154], an FE array has been used that contains 27 electrodes to deliver the FES stimulation (figure 7). The overall system comprises a kinematic glove, EMG unit, and FES module. The stimulation configuration of FES electrodes is automatically selected based on the feedback received from the kinematic glove (records finger and wrist movements) and EMG (records forearms muscles activity). The system has been tested on eight healthy subjects to perform different finger and wrist movements of left arm. Results show that the electrode array successfully provides an accurate stimulation to targeted muscles and could be feasible for stroke rehabilitation applications. Also, in [155], a flexible on-skin 80 electrode array has been developed for controlling FES stimulation with EMG feedback. At a single time, 20 electrodes can be activated via switching logic in order to provide the required stimulation to lower arm muscles for fine movement control (figure 8). For performance evaluation, the overall device containing FES and EMG measurement unit is under development phase. Yang et al [181] fabricated the screen-printed fabric electrode arrays (FEA), containing 24 electrodes for wearable FES device (figure 12(a)). First, optimized stimulation sites on the forearm are selected, and then FES stimulation is delivered to the targeted location via FEA. Results show the successful execution of desired movements, including 'open hand', 'pinch' and 'pointing' gestures (figure 12(b)). Hence, it can be used in stroke rehabilitation systems to provide upper limb rehabilitation therapies. Another multi-pad flexible electrode array for FES stimulation was designed by Malešević et al [182], which is named intelligent functional electrical stimulation (INTFES). The array is made of a flexible polyester substrate and contains 16 electrodes that can be controlled individually. The system has been tested on three stroke survivors where the electrodes are placed on forearm muscles for producing grasping movements. The selective activation of electrodes is based on flex sensors feedback that measures the muscle twitch response and automatically activates the electrodes accordingly (figure 13). The result shows that the INTFES triggers the correct electrode configuration and successfully accomplishes grasping action along with maintaining wrist stabilization. Similarly, Loitz et al [183] also developed multi-pad FEA for FES control in which the electrode activation is controlled by flex sensors feedback (figures 14(a) and (b)). The system has been designed specifically for stroke rehabilitation purposes and has been successfully tested on a stroke survivor to perform hand opening.

\subsubsection{Robotics based rehabilitation/assistive systems}

The main advantage of robotics systems over other methods in stroke rehabilitation is their possible application to subjects with extremely low or even no motor function. Many researches have shown that the robotics rehabilitation methods produce an improved stroke recovery compared to the conventional rehabilitation approaches [187]. Furthermore, the flexible design introduces flexibility that allows the subject to perform rehabilitative movements with a higher range of motion and greater ease. Despite having several advantages, robotics rehabilitation systems have limitations in terms of their massive, rigid and complex operating setup, making subjects uncomfortable and less motivated towards performing rehabilitation exercises [35, 63, 188-193]. Thus, to develop compact and flexible robotics system, the use of FT via 'Soft Robotics' comes into play. Using stretchable materials and flexible actuators, soft robotics has introduced a new paradigm for human-machine applications and has successfully been demonstrated its adaptability [194-196], agility [107, 197, 198], and sensitivity [199, 200].

Currently, the application of soft robotics in stroke robotics systems is at its rising curve and several studies have reported their implementation in assistive and rehabilitative robotics for regaining the subject's movement and motor recovery [201-203]. For post-stroke gait rehabilitation, a textile based flexible wearable robotic system has been developed $[201,202]$ that transmits the actuator's generated power to the paretic ankle and provides assistance during walking (figure 15). The system has been tested on nine stroke survivors (chronic phase), and results show that the paretic limb achieved an increase in ankle's swing phase dorsiflexion and improved forward propulsion. In [203], as a part of the European project 'XoSoft', a soft exoskeleton (exosuits) has been developed to assist people having mobility pathologies (figure 16). This system assists in people walking 
(a)

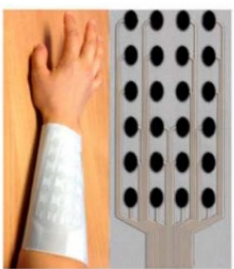



Figure 12. (a) Flexible electrode array (b) stimulation patterns for pointing, pinching and open hand gestures. Reprinted from [181], Copyright (2014), with permission from Elsevier.



Figure 13. Sensor system for the assessment of the effects of stimulation. The flex sensors are affixed with special rings, and the glove is applied using a zipper interface. The electronics for processing are integrated into the system. The outputs are finger flexion/extension and wrist flexion/extension movements. Reprinted by permission from Springer Nature Customer Service Centre GmbH: Springer Nature, Journal of neuroengineering and rehabilitation [182] A multi-pad electrode based functional electrical stimulation system for restoration of grasp, Malešević et al, (c) 2012.

and provides mechanical assistance by reducing the energy requirements within a range of $10 \%-20 \%$. The performance of the developed exosuit has been evaluated on stroke survivors in a straight walking scenario. It has been found that the exoskeleton provides power assistance of $9.3 \pm 3.5 \%$ and $10.9 \pm 2.2 \%$ for knee and hip actuation, respectively. Additionally, improvement in gait pattern and increased foot clearance has also been observed at different phases of the gait cycle. In another research, Bae et al [204] developed a lightweight and efficient portable soft exosuit for paretic ankle assistance during gait rehabilitation. A preliminary testing has been performed on 03 stroke patients and it demonstrates that the soft exosuit can improve paretic limb ground clearance and forward propulsion, hence, decreasing the metabolic cost of walking. Awad et al [205] presented a soft robotic exosuit (figure 17) and evaluate its effects on the long and short distance walking ability of stroke patients. The soft rehabilitation exosuit has been tested on six stroke patients who are in the chronic phase of post-stroke recovery. The findings report that a portable soft exosuit facilitates the farther walking distances and faster walking speeds among the stroke individuals. For post-stroke hand rehabilitation, Stilli et al [206] designed a novel light-weight inflatable soft exoskeleton device, called the AirExGlove. It delivers adaptive, high-dosage and gradual rehabilitation to the stroke patients affected by clenched fist deformity. Preliminary testing of AirExGlove on clenched-fist stroke patient validates a higher level of ergonomics of the soft exoskeleton in comparison with conventional robotic systems. Another soft robotic glove for upper limb rehabilitation has been developed by Yap et al [207] that provides grasping assistance via promoting finger flexion (figure 18). Pilot testing has been performed on two stroke patients that confirms the improvement in patient's grasping action. Recently, Cheng et al [208] investigates the clinical application of BCI based soft robotic glove (BCI-SRG). The randomized controlled feasibility study is performed on 11 chronic stroke patients and it is found that BCISRG group depicts the trends of prolonged improvements in rehabilitation scores.'

\subsubsection{Overview of stroke rehabilitation and assistive devices}

Table 2 shows an overview of different studies in which FT has been used to develop FES rehabilitation devices and soft robotics-based rehabilitation/assistive systems.

\section{Discussion}

The word 'Flexible Technology' itself is a much diversified term and possesses a wide variety of medical applications. Currently, FT is quickly emerging in research activities to provide novel healthcare solutions for the stroke community (figure 19). FT mainly includes the implementation of FE, E-textile, and Soft Robotics to develop the systems for post-stroke use. These systems are regarded as rehabilitation and assistive systems, which fall under the two broad categories. The first one is entirely operated via allocated hardware units, whereas the second one is controlled via voluntary intention of users (by using their EEG or EMG signals). Thus, exhaustive research is underway for developing biosignal acquisition electrodes/systems, assistive devices, and rehabilitation systems via flexible technology. FT is currently in its early stages of development; however, preliminary 
(a)

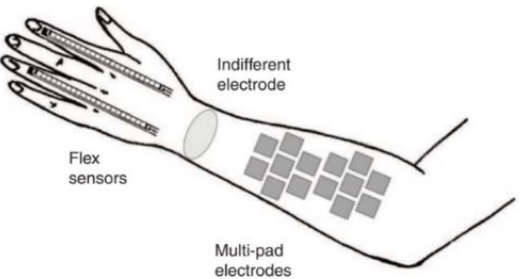

(b)

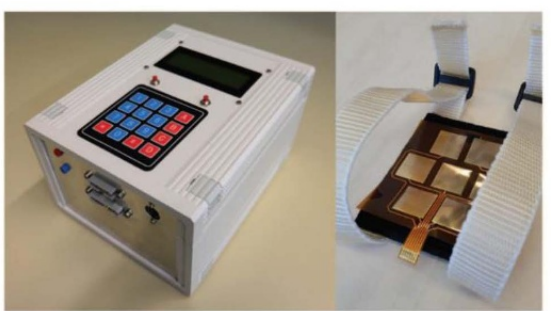

Figure 14. (a) Schematic illustration of multi-pad electrode FES with sensor feedback. (b) Left: multi-pad control hardware with front panel to connect electrodes and sensors. Right: custom-made multi-pad electrode and sleeve. Reproduced with permission from [183].

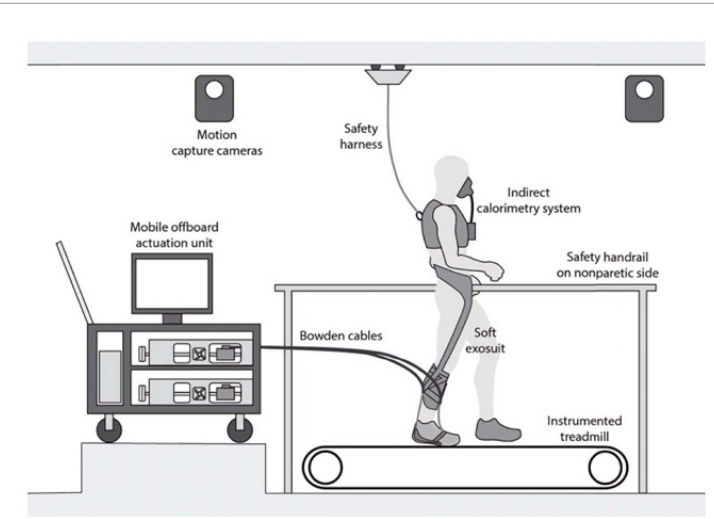

Figure 15. Illustration of experimental setup. Instrumented treadmill with the exosuit worn either powered (delivering forces generated by an off-board actuation unit) or unpowered. From [201]. Reprinted with permission from AAAS.

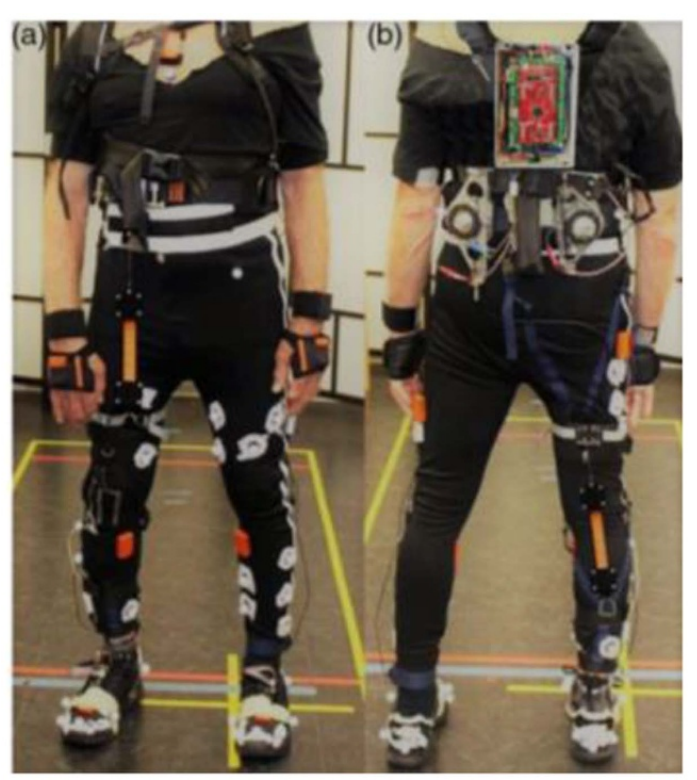

Figure 16. Participant wearing XoSoft: (a) front and (b) back view. Reproduced with permission from [203].

results are quite promising and provide the path for future innovation. In addition to the detailed explanation of FT's stroke application, we believe that some

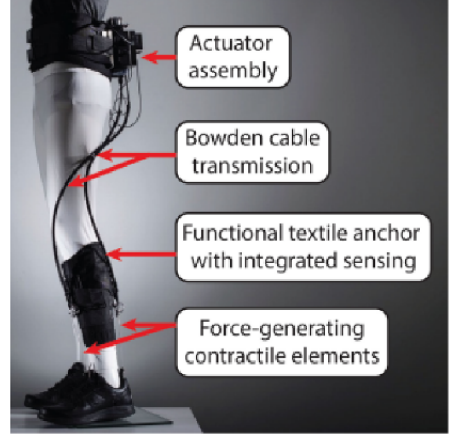

Figure 17. Overview of the soft robotic exosuit used to augment paretic ankle plantarflexion (PF) and dorsiflexion (DF) function during post-stroke hemiparetic walking. (C) [2020] IEEE. Reprinted, with permission, from [205].

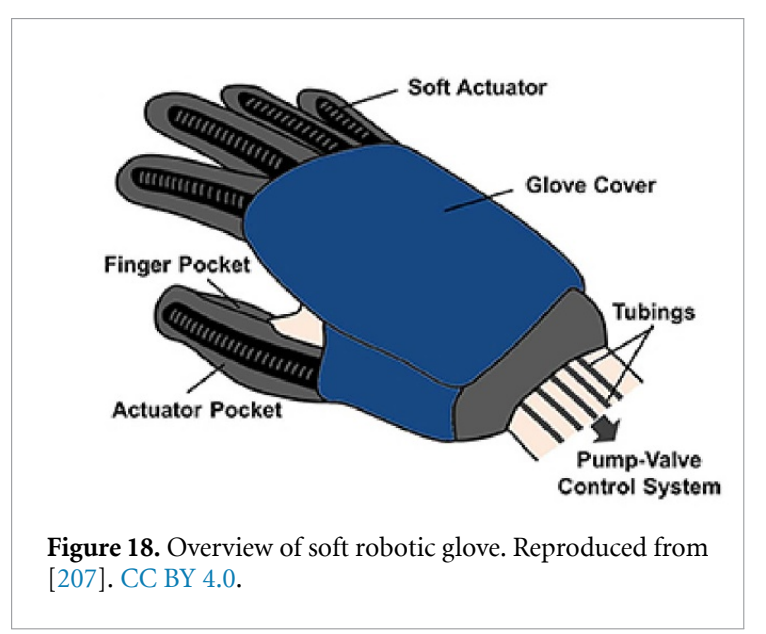

key questions need to be addressed for concluding the discussion. These queries can provide an overview regarding the advantages, limitations, and further interpretation of FT in stroke systems.

\subsection{What are the key advantages of FT in post-stroke systems?}

The key advantages of FT in developing medical systems for stroke patients vary based on their application mode and are comprehensively described below. 
Table 2. Research studies and their outcomes for the development of stroke rehabilitation and assistive devices.

\begin{tabular}{|c|c|c|}
\hline Study & Designed Hardware & Post-Stroke Rehab Applications \\
\hline Yang et al [180] & $\begin{array}{l}\text { 'e-sleeve' (flexible } 24 \text {-electrode } \\
\text { array) (Fabricated by Screen } \\
\text { printing [167]) }\end{array}$ & $\begin{array}{l}\text { Tested on eight stroke patients } \\
\text { for applying FES stimulation to } \\
\text { perform upper limb } \\
\text { rehabilitation. }\end{array}$ \\
\hline $\begin{array}{l}\text { De Marchis et al } \\
{[154]}\end{array}$ & Flexible 27 electrode array & $\begin{array}{l}\text { Pilot testing is performed on } \\
\text { eight healthy subjects for } \\
\text { applying FES stimulation. } \\
\text { Hence, shows the potential for } \\
\text { post-stroke rehabilitation. }\end{array}$ \\
\hline Duente et al [155] & Flexible 80 electrode array & $\begin{array}{l}\text { Providing FES stimulation to } \\
\text { lower arm for post-stroke } \\
\text { rehabilitation. }\end{array}$ \\
\hline Yang et al [181] & $\begin{array}{l}\text { Fabric electrode arrays (FEA), } \\
\text { containing } 24 \text { electrodes } \\
\text { (Fabricated by Screen printing } \\
{[167] \text { ) }}\end{array}$ & $\begin{array}{l}\text { Pilot testing is performed on } \\
\text { two healthy subjects for } \\
\text { applying FES stimulation. } \\
\text { Hence, shows the potential for } \\
\text { post-stroke rehabilitation. }\end{array}$ \\
\hline
\end{tabular}

Malešević et al [182] Multi-pad electrode based system-INTFES (INTelligent Functional Electrical Stimulation)

Loitz et al [183] Multi-pad electrode (Fabricated by Flexible printed circuit (FPC) technology

Awad et al [201] and Textile based flexible wearable Bae et al [202]

Natali et al [203]

Soft lower limb exoskeletons (exosuits)

Bae et al [204]

Soft robotics exosuit

Award et al [205]

Soft robotics exosuit

Stilli et al [206]

Soft pneumatic exoskeleton glove (AirExGlove)

Yap et al [207]

Soft Robotic Glove

Cheng et al [208]
Tested on three stroke patients for delivering FES stimulation to a forearm during upper limb rehabilitation.

Tested on single stroke patient for applying FES stimulation to perform upper limb rehabilitation.

Tested on nine stroke patients for gait rehabilitation
System perfectly activated the optimized electrodes for carrying out 'hand opening and pointing' gestures.

System perfectly activated the optimized electrodes for carrying out hand wrist movements.

20 electrodes can be activated at a single time. FES and EMG unit is under development for future testing.

Successfully executed 'open hand', 'pinch', and 'pointing' gestures. FEA Performance is compared with plastic electrode array. Result showed the achievement of higher angular joint movement and greater repeatability by using FEA.

INTFES triggered the correct electrode configuration and successfully stimulates the muscles required for grasping.

Provided stimulation to extensor digitorium muscle for accomplishing hand opening

Increase in ankle's swing phase dorsiflexion, and improved forward propulsion has been achieved.

Performance evaluation is made Improvement in gait pattern on one post-stroke patient for gait assistance during rehabilitation.

Tested on 03 stroke patient for paretic ankle assistance during gait rehabilitation.

Tested on 06 chronic stroke patients for ankle assistance during gait rehabilitation.

Preliminary testing of AirExGlove is performed on clenched-fist stroke patient for hand rehabilitation. Preliminary testing of glove is performed on 02 stroke patient for hand rehabilitation.

and increased foot clearance has been observed

Analysis showed the improved paretic limb ground clearance and forward propulsion during walking.

Facilitated the farther walking distances and faster walking speeds among the stroke individuals.

Showed higher level of ergonomics in comparison with conventional rehabilitation robots.

Pilot testing confirmed the improvement in patient's grasping performance. BCI-SRG group showed more improvements in rehabilitation scores as compared to SRG control group. stroke patients to assess their performance in comparison with 'without BCI' soft robotic glove. 


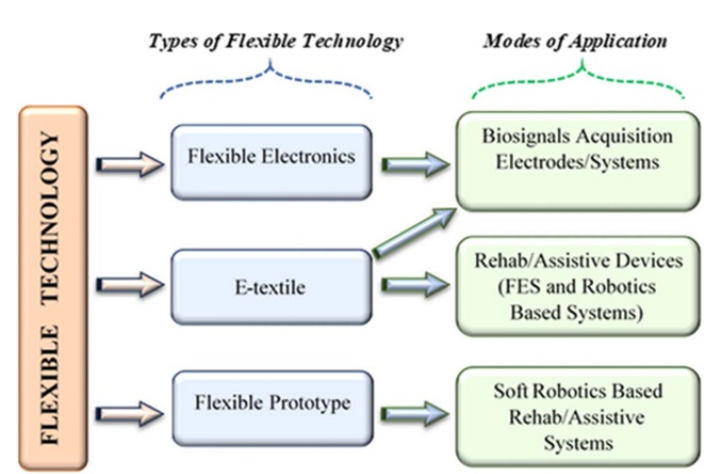

Figure 19. Overview of types of flexible technology and their modes of application.

\subsubsection{FE based electrodes and systems}

FE plays a vital role in developing biosignal acquisition electrodes (e.g. EEG, EMG, etc and healthcare systems (e.g. FES device, biosensors, etc). The use of FE over the traditional approaches allows the system to be more compact and portable, enabling the user to perform long-lasting rehabilitation exercises or other similar activities with a high level of comfort and ease [126]. Additionally, the FAEs eliminate the need for complex wiring, reducing the movement artifacts and recording the clean signals compared to lead-based systems. Such adhesive electrodes are also very useful for emergency medical care in ambulance work due to their 'disposability' nature. It also provides an opportunity to place the flexible module where the rigid component is challenging to place. For instance, the placement of flexible EEG sensors on the earlobe for measuring brain signals [139]. Moreover, it allows the quick and easy installation of a flexible system in case of an emergency that includes the usage of EEG headset in case of emergency for continuous monitoring of brain condition [138].

\subsubsection{E-textile}

The concept of e-textile (also termed as 'Smart Textile') is purely based on the concept of FT and is seen as an innovative way to revolutionize healthcare practices. The most important advantage that brings the smart textile in the high-demand list is the introduction of 'Customized Wearability'. As different electronic modules can be embedded into any clothing, a wearable system becomes more adaptable. The user can personalize its appearance depending on individual preferences and environmental changes. Furthermore, instead of using physical electronic components, some of the e-textile contain the printed modules that are printed on the stretchable textiles via conductive inks. This reduces the weight and improves its wearability, which converts the normal textile into an intelligent textile that can compute, sense, actuate and communicate (depending on the application). Within the post-stroke systems, e-textile contains a wide variety of applications, which mainly comprises fabrication of EEG electrodes [141, 142], FES stimulation electrodes [180-182], and gait rehabilitation systems [201, 202].

\subsubsection{Flexible prototype for soft robotics based assistive/rehabilitation unit}

In such systems, FT comes into action via 'Soft Robotics' that provides a high DOF and more flexibility while performing mobility action. Moreover, flexible exoskeletons and orthosis show promising results especially because of the reduced weight that is crucial when performing rehabilitation therapy. Two major limitations of the rigid exoskeleton that prevent it from home/community use are high cost and the requirement of expertise to operate. One of the advantages of flexible sensors and e-textile system application in soft wearable robotics is that it could potentially bring down the cost and requires less expertise to operate, which therefore makes it possible for long-term home use.

\subsection{What are the limitations of FT for the development of post-stroke systems?}

The limitation of FT varies depending on its mode of application.

\subsubsection{FE}

The primary constraint in FE-based systems/electrodes is 'Mechanical Robustness,' i.e. the flexible components started to lose their stability and behave differently after few cycles of twisting, stretching, and bending [209]. Among flexible adhesive sensors/electrodes, the key limitation is their 'Re-usability,' i.e. depending on the adhesion quality, the adhesive sensor needs to be replaced with a new one after few trials [210]. Another disadvantage of FE-based skin sensors is 'Unstable Output due to Motion Artifacts.' The skin stretching causes variations in the skin potential, which can affect the sensor output and lead to generation of false readings [211]. Some people consider 'Hair Removal' as a weakness of FE. To obtain high-quality biosignals via flexible sensors, the targeted area must be properly shaved to avoid interference caused by hair [132]. Another drawback includes the 'Difficulty of Repairing or Modifying' the FE components in the system. Hence, great care must be taken while designing and fabricating the flexible modules.

\subsubsection{E-textile}

'Washability' is the main obstacle that limits the practical implementation of e-textile in healthcare. Currently, there are no standardized washing protocols available for e-textiles, and every e-textile manufacturer provides its own reliability statements for repeated washing [212]. The washing process might produce any or all of the following effects in the respective e-textile: 
- Changes in the electrical properties of the conductive path: For instance, increase in the resistance or the loss of electrical conductivity, etc [213-215].

- Changes in integrity: For instance, loosening of components/wires, delamination, etc [216, 217].

- Changes in the textile characteristics: For instance, decreased sensing capabilities, reduced data transmission ranges, etc $[218,219]$.

- Mechanical deformation: For instance, the appearance of wrinkles in the fabrics that introduce artifacts in the sensing measurements, etc [220].

- Functional changes: Partial or complete functional loss of the e-textile system [221, 222].

\subsubsection{Soft robotics based assistive/rehabilitation unit}

Among soft robotics based rehabilitation systems, the hydraulic and pneumatic control mechanism are widely used. Such control strategies require high pressure pumps for their actuation that causes difficulties in retaining the required gripping force and therefore, result in slow actuation rates [223]. Moreover, soft robotics in rehabilitation robots often offers a lower number of DOFs as compared to classical rigid robotics systems [224]. Hence, in future design, these limitations should be taken into consideration to further increase the adaption of soft robotics in stroke rehabilitation.

\subsection{Is FT used in any post-stroke system regarding the invasive and semi-invasive application?}

Invasive/semi-invasive application is the best suitable ones for biosignals acquisition modules within the rehab and assistive systems. Hence, it is expected that FE-based invasive/semi-invasive biosignal acquisition systems will be developed in the future. The in-vivo experiments have already been performed on feline animal (cat) models to acquire their brain signals via a semi-invasive flexible ECoG sensing unit [225, 226]. The preliminary results show that flexible semiinvasive electrodes have great potential for measuring high-quality brain activities. However, it possesses several limitations, including [227]: (a) These electrodes need the surgical procedure for implantation. (b) There are chances that the body will not respond to the new external object as expected, thus, causing medical problems. (c) Complications related to the implant stability and possible neural infection can also arise. Therefore, their feasibility for human trials is still questionable, and thus, the current usage of FE in an invasive recording is restricted for post-stroke applications.

\subsection{In addition to the biosignal measurement and rehabilitation/assistive systems, is there any other possible implementation of FT in stroke application?}

For most patients with stroke, the amount of rehabilitation provided starts decreasing once they leave the hospital. Hence, despite being a great technology for neurorehabilitation acquisition systems and devices, FT can also be used in developing sensors to provide accurate and long-term measurements of several vital parameters that healthcare professionals can remotely analyze. Recent work from Lee et al [228] presents the development of a flexible skin-mounted sensing device. It incorporates high-bandwidth triaxial accelerometers and is placed at the suprasternal notch (a visible dip in between the neck). The device can provide real-time recordings of respiration rate, swallowing count, talking time, body orientation, heart rate, and sleep quality. In [229], Kim et al developed an e-skin (electronic skin) integrated electronic system that can perform wireless sensing of voice, breathing, pulse wave, chewing/swallowing, temperature, and knee movements. The acquired data is transmitted via Bluetooth unit to the smartphone for real-time display. Also, Murphy et al [230] designed an upper-body flexible garment with integrated sensors that are able to monitor heart rate and movement-related parameters to evaluate the progression in post-stroke recovery. Hence, such kinds of intermodal sensing systems could be of great importance in post-stroke applications. They can send an overview of the patient's physiological state to the clinician's phones and computers. Consequently, the clinician can take the necessary steps at the right time, which could lead to faster and better recoveries for stroke patients [209].

\section{Conclusion}

The analysis of post-stroke function is a long process, starting from impairment assessment, rehabilitative training, monitoring recovery, and providing required assistance to perform everyday life activities. With every passing day, the modern era of technological advancement allows to develop new and innovative post-stroke systems with an aim to support the stroke community. Among them, FT is on the rising curve that provides novel solutions to develop compact, portable, user-friendly, lightweight, and wearable healthcare systems for stroke patients. Hence, in this review, different types of FT for developing stroke systems have been presented, including FE, e-textile, and flexible prototype. It has been shown that every FT has its specific application in developing different modules involved in post-stroke systems. FE is widely used in biosignal acquisition electrodes/units. Similarly, e-textile is adopted for developing various electrodes (EEG, EMG, and FES) and assistive devices. On the other hand, a flexible prototype is mainly used to develop soft robotics systems for advanced rehabilitation and assistive purposes. Every technology has its own advantages and limitations that have been described in detail in the manuscript. Finally, the other possible stroke application of FT and its future perspective has also been discussed that can 
further revolutionize the development of upcoming post-stroke medical systems.

\section{Data availability statement}

All data that support the findings of this study are included within the article (and any supplementary files).

\section{Acknowledgments}

The research leading to these results has received funding from the European Union's Horizon 2020 research and innovation programme under the Marie Sklodowska-Curie Grant Agreement No. 713683 (COFUNDfellowsDTU)

\section{ORCID iDs}

Muhammad Ahmed Khan (1) https://orcid.org/00000002-8696-4227

Rig Das (1) https://orcid.org/0000-0001-9463-4298 Iris Brunner (i) https://orcid.org/0000-0002-71943087

Sadasivan Puthusserypady (] https://orcid.org/00000001-7564-2612

\section{References}

[1] Robinson R G and Jorge R E 2016 Post-stroke depression: a review Am. J. Psychiatry 173 221-31

[2] Khedr E M et al 2020 Post-stroke depression: frequency, risk factors, and impact on quality of life among 103 stroke patients—hospital-based study Egyptian J. Neurol. Psychiatry Neurosurg. 56 1-8

[3] Feigin V L et al 2015 Update on the global burden of ischemic and hemorrhagic stroke in 1990-2013: the GBD 2013 study Neuroepidemiology 45 161-76

[4] Johnson C O et al 2019 Global, regional, and national burden of stroke, 1990-2016: a systematic analysis for the Global Burden of Disease Study 2016 Faculty Opin.-Post-Pub. Peer Rev. Biomed. Literat. 18 439-58

[5] Ballester B R et al 2019 A critical time window for recovery extends beyond one-year post-stroke J. Neurophysiol. $122350-57$

[6] Bernhardt J et al 2017 Agreed definitions and a shared vision for new standards in stroke recovery research: the stroke recovery and rehabilitation roundtable taskforce Int. J. Stroke 12 444-50

[7] Page S J, Levine P, Sisto S and Johnston M V 2001 A randomized efficacy and feasibility study of imagery in acute stroke Clin. Rehabil. 15 233-40

[8] Claflin E S, Krishnan C and Khot S P 2015 Emerging treatments for motor rehabilitation after stroke Neurohospitalist 5 77-88

[9] Birbaumer N, Ruiz S and Sitaram R 2013 Learned regulation of brain metabolism Trends Cogn. Sci. 17 295-302

[10] Ruiz S, Buyukturkoglu K, Rana M, Birbaumer N and Sitaram R 2014 Real-time fMRI brain computer interfaces: self-regulation of single brain regions to networks Biol. Psychol. 95 4-20

[11] Khan M A, Das R, Iversen H K and Puthusserypady S 2020 Review on motor imagery based BCI systems for upper limb post-stroke neurorehabilitation: from designing to application Comput. Biol. Med. 123103843

[12] Eraifej J et al 2017 Effectiveness of upper limb functional electrical stimulation after stroke for the improvement of activities of daily living and motor function: a systematic review and meta-analysis Syst. Rev. 6 1-21

[13] Da Cunha M J et al 2020 Functional electrical stimulation of the peroneal nerve improves post-stroke gait speed when combined with physiotherapy. A systematic review and meta-analysis Ann. Phys. Rehabil. Med. 64101388

[14] Khan M A, Abdullah S, Serpelloni M and Sardini E 2018 Design of FES based muscle stimulator device using EMG and insole force resistive sensors for foot drop patients $A d v$. Mater. Lett. $9776-80$

[15] Moe J and Post H 1962 Functional electrical stimulation for ambulation in hemiplegia J. Lancet. 82285

[16] Popovic M R, Thrasher T A, Adams M E, Takes V, Zivanovic V and Tonack M I 2006 Functional electrical therapy: retraining grasping in spinal cord injury Spinal Cord 44 143-51

[17] Grill J H and Peckham P H 1998 Functional neuromuscular stimulation for combined control of elbow extension and hand grasp in C5 and C6 quadriplegics IEEE Trans. Rehabil. Eng. 6 190-9

[18] Ethier C et al 2012 Restoration of grasp following paralysis through brain-controlled stimulation of muscles Nature $485368-71$

[19] Daly J J et al 2011 Recovery of coordinated gait: randomized controlled stroke trial of functional electrical stimulation (FES) versus no FES, with weight-supported treadmill and over-ground training Neurorehabil. Neural Repair 25 588-96

[20] Alon G, Levitt A F and McCarthy P A 2008 Functional electrical stimulation (FES) may modify the poor prognosis of stroke survivors with severe motor loss of the upper extremity: a preliminary study Am. J. Phys. Med. Rehabil. 87 627-36

[21] Ajiboye A B et al 2017 Restoration of reaching and grasping movements through brain-controlled muscle stimulation in a person with tetraplegia: a proof-of-concept demonstration Lancet 389 1821-30

[22] Popovic M B, Popovic D B, Sinkjaer T, Stefanovic A and Schwirtlich L 2002 Restitution of reaching and grasping promoted by functional electrical therapy Artif. Organs. $26271-5$

[23] Nataraj R, Audu M L, Kirsch R F and Triolo R J 2010 Comprehensive joint feedback control for standing by functional neuromuscular stimulation-a simulation study IEEE Trans. Neural. Syst. Rehabil. Eng. 18 646-57

[24] Nataraj R, Audu M L and Triolo R J 2017 Restoring standing capabilities with feedback control of functional neuromuscular stimulation following spinal cord injury Med. Eng. Phys. 42 13-25

[25] Howlett O A, Lannin N A, Ada L and McKinstry C 2015 Functional electrical stimulation improves activity after stroke: a systematic review with meta-analysis Arch. Phys. Med. Rehabil. 96 934-43

[26] Tavella $\mathrm{M}$ et al 2010 Towards natural non-invasive hand neuroprostheses for daily living 2010 Annual Int. Conf. IEEE Engineering in Medicine and Biology (IEEE) (https:// doi.org/10.1109/IEMBS.2010.5627178)

[27] Excell T et al 2013 Optimisation of hand posture stimulation using an electrode array and iterative learning control J. Autom. Control 21 1-4

[28] Wang S et al 2014 Design and control of the MINDWALKER exoskeleton IEEE Trans. Neural. Syst. Rehabil. Eng. 23 277-86

[29] Sankaranarayan $\mathrm{H}$ et al 2016 Role of ankle foot orthosis in improving locomotion and functional recovery in patients with stroke: a prospective rehabilitation study J. Neurosci. Rural Pract. 7544 
[30] Shrivastava S et al 2014 Ankle foot orthosis for walking in stroke rehabilitation Cochrane Database Syst. Rev. 8 CD011249

[31] Farrell J F, Hoffman H B, Snyder J L, Giuliani C A and Bohannon R W 2007 Orthotic aided training of the paretic upper limb in chronic stroke: results of a phase 1 trial Neuro Rehabil. 22 99-103

[32] Tyson S F and Kent R M 2011 The effect of upper limb orthotics after stroke: a systematic review Neuro Rehabil. 28 29-36

[33] Ambrosini E et al 2019 Upper-limb exoskeletons for stroke rehabilitation Mediterranean Conf. on Medical and Biological Engineering and Computing (Springer)

[34] Meng Q et al 2019 Pilot study of a powered exoskeleton for upper limb rehabilitation based on the wheelchair Biomed. Res. Int. 20199627438

[35] Xu B et al 2015 Robotic neurorehabilitation system design for stroke patients Adv. Mech. Eng. 71687814015573768

[36] Sarac M, Ergin M A and Patoglu V 2013 AssistOn-Mobile: a series elastic holonomic mobile platform for upper extremity rehabilitation 2013 World Haptics Conf. (WHC) (IEEE) 283-8

[37] Pehlivan A U, Celik O and O’Malley M K 2011 Mechanical design of a distal arm exoskeleton for stroke and spinal cord injury rehabilitation 2011 IEEE Int. Conf. on Rehabilitation Robotics (IEEE) (https://doi.org/ 10.1109/ICORR.2011.5975428)

[38] Cantillo-Negrete J et al 2015 Control signal for a mechatronic hand orthosis aimed for neurorehabilitation 2015 Pan American Health Care Exchanges (PAHCE) (IEEE) (https://doi.org/10.1109/PAHCE.2015.7173328)

[39] Abdullah S, A. Khan M, Serpelloni M and Sardini E 2019 Hybrid EEG-EMG based brain computer interface (BCI) system for real-time robotic arm control Adv. Mater. Lett. $1035-40$

[40] Timmermans A A et al 2009 Technology-assisted training of arm-hand skills in stroke: concepts on reacquisition of motor control and therapist guidelines for rehabilitation technology design J. Neuroeng. Rehabil. 6 1-18

[41] Machado T C, Carregosa A A, Santos M S, Ribeiro N M D S and Melo A 2019 Efficacy of motor imagery additional to motor-based therapy in the recovery of motor function of the upper limb in post-stroke individuals: a systematic review Top. Stroke Rehabil. 26 548-53

[42] Johnson S H, Sprehn G and Saykin A J 2002 Intact motor imagery in chronic upper limb hemiplegics: evidence for activity-independent action representations J. Cogn. Neurosci. 14 841-52

[43] Sharma N, Pomeroy V M and Baron J-C 2006 Motor imagery: a backdoor to the motor system after stroke? Stroke 37 1941-52

[44] Das R et al 2020 FBCSP and adaptive boosting for multiclass motor imagery BCI data classification: a machine learning approach 2020 IEEE Int. Conf. on Systems, Man, and Cybernetics (SMC) (IEEE) (https://doi. org/10.1109/SMC42975.2020.9283098)

[45] Braun S, Kleynen M, van Heel T, Kruithof N, Wade D and Beurskens A 2013 The effects of mental practice in neurological rehabilitation; a systematic review and meta-analysis Front. Hum. Neurosci. 7390

[46] Dickstein R and Deutsch J E 2007 Motor imagery in physical therapist practice Phys. Ther. 87 942-53

[47] Jure F A, Carrere L C, Gentiletti G G and Tabernig C B 2016 BCI-FES system for neuro-rehabilitation of stroke patients J. Phys.: Conf. Ser. 705012058

[48] Daly J J, Cheng R, Rogers J, Litinas K, Hrovat K and Dohring M 2009 Feasibility of a new application of noninvasive brain computer interface (BCI): a case study of training for recovery of volitional motor control after stroke J. Neurol. Phys. Ther. 33 203-11

[49] Leeb R et al 2010 On the road to a neuroprosthetic hand: a novel hand grasp orthosis based on functional electrical stimulation 2010 Annual Int. Conf. IEEE Engineering in
Medicine and Biology (IEEE) (https://doi.org/ 10.1109/IEMBS.2010.5627412)

[50] Pfurtscheller G, Müller G R, Pfurtscheller J, Gerner H J and Rupp R 2003 'Thought'-control of functional electrical stimulation to restore hand grasp in a patient with tetraplegia Neurosci. Lett. 351 33-36

[51] Cincotti F et al 2012 EEG-based brain-computer interface to support post-stroke motor rehabilitation of the upper limb 2012 Annual Int. Conf. IEEE Engineering in Medicine and Biology Society (IEEE) (https://doi.org/ 10.1109/EMBC.2012.6346871)

[52] Li M et al 2014 Neurophysiological substrates of stroke patients with motor imagery-based brain-computer interface training Int. J. Neurosci. 124 403-15

[53] Mukaino M, Ono T, Shindo K, Fujiwara T, Ota T, Kimura A, Liu M and Ushiba J 2014 Efficacy of brain-computer interface-driven neuromuscular electrical stimulation for chronic paresis after stroke J. Rehabil. Med. $46378-82$

[54] Kim T, Kim S and Lee B 2016 Effects of action observational training plus brain-computer interface-based functional electrical stimulation on paretic arm motor recovery in patient with stroke: a randomized controlled trial Occup. Ther. Int. 23 39-47

[55] Tabernig C B et al 2018 Neurorehabilitation therapy of patients with severe stroke based on functional electrical stimulation commanded by a brain computer interface $J$. Rehabil. Assis. Technol. Eng. 52055668318789280

[56] Sabathiel N et al 2016 Paired associative stimulation with brain-computer interfaces: a new paradigm for stroke rehabilitation Int. Conf. on Augmented Cognition (Springer) (https://doi.org/10.1007/978-3-319-39955-3_25)

[57] Cho W, Heilinger A, Xu R, Zehetner M, Schobesberger S, Murovec N, Ortner R and Guger C 2017 Hemiparetic stroke rehabilitation using avatar and electrical stimulation based on non-invasive brain computer interface Int. J. Phys. Med. Rehabil. 52

[58] Qiu Z et al 2018 BCI-based strategies on stroke rehabilitation with avatar and FES feedback (arXiv:1805.04986)

[59] Irimia D-C et al 2017 Preliminary results of testing a BCI-controlled FES system for post-stroke rehabilitation GBCIC (https://doi.org/10.3217/978-3-85125-533-1-38)

[60] Varkuti B et al 2013 Resting state changes in functional connectivity correlate with movement recovery for BCI and robot-assisted upper-extremity training after stroke Neurorehabil. Neural Repair 27 53-62

[61] Ang K K et al 2015 A randomized controlled trial of EEG-based motor imagery brain-computer interface robotic rehabilitation for stroke Clin. EEG Neurosci. $46310-20$

[62] Ang K K et al 2014 Brain-computer interface-based robotic end effector system for wrist and hand rehabilitation: results of a three-armed randomized controlled trial for chronic stroke Front. Neuroeng. 730

[63] Sarac M et al 2013 Brain computer interface based robotic rehabilitation with online modification of task speed 2013 IEEE 13th Int. Conf. on Rehabilitation Robotics (ICORR) (IEEE) (https://doi.org/10.1109/ICORR.2013.6650423)

[64] Bhagat N A et al 2014 Detecting movement intent from scalp EEG in a novel upper limb robotic rehabilitation system for stroke 201436 th Annual Int. Conf. IEEE Engineering in Medicine and Biology Society (IEEE) (https:// doi.org/10.1109/EMBC.2014.6944532)

[65] Frolov A A et al 2017 Post-stroke rehabilitation training with a motor-imagery-based brain-computer interface (BCI)-controlled hand exoskeleton: a randomized controlled multicenter trial Front. Neurosci. 11400

[66] Cantillo-Negrete J et al 2018 Motor imagery-based brain-computer interface coupled to a robotic hand orthosis aimed for neurorehabilitation of stroke patients $J$. Healthc Eng. 20181624637 
[67] Olesen S D T et al 2021 Hybrid EEG-EOG-based BCI system for vehicle control 2021 9th Int. Winter Conf. on Brain-Computer Interface (BCI) (IEEE) (https://doi.org/ 10.1109/BCI51272.2021.9385300)

[68] Wang Y, Gao X, Hong B O, Jia C and Gao S 2008 Brain-computer interfaces based on visual evoked potentials IEEE Eng. Med. Biol. Mag. 27 64-71

[69] Ng D W-K and Goh S Y 2020 Indirect control of an autonomous wheelchair using SSVEP BCI J. Robot. Mechatron. 32 761-7

[70] Mistry K S et al 2018 An SSVEP based brain computer interface system to control electric wheelchairs 2018 IEEE Int. Instrumentation and Measurement Technology Conf. (I2MTC) (IEEE) (https://doi.org/10.1109/I2MTC. 2018.8409632)

[71] Singla R, Khosla A and Jha R 2014 Influence of stimuli colour in SSVEP-based BCI wheelchair control using support vector machines J. Med. Eng. Technol. 38 125-34

[72] Turnip A, Suhendra M A and Ws M S 2015 Brain-controlled wheelchair based EEG-SSVEP signals classified by nonlinear adaptive filter 2015 IEEE Int. Conf. on Rehabilitation Robotics (ICORR) (IEEE) (https://doi. org/10.1109/ICORR.2015.7281318)

[73] Piyus C K, Cherian V A and Nageswaran S 2017 EMG based FES for post-stroke rehabilitation IOP Conf. Ser. Mater. Sci. Eng. 263052025

[74] Monte-Silva K et al 2019 Electromyogram-related neuromuscular electrical stimulation for restoring wrist and hand movement in poststroke hemiplegia: a systematic review and meta-analysis Neurorehabil. Neural Repair 33 96-111

[75] Hara Y 2013 Rehabilitation with functional electrical stimulation in stroke patients Int. J. Phys. Med. Rehabil. 12

[76] Oonishi Y, Oh S and Hori Y 2010 A new control method for power-assisted wheelchair based on the surface myoelectric signal IEEE Trans. Ind. Electron. 57 3191-6

[77] Felzer T and Freisleben B 2002 HaWCoS: the "hands-free" wheelchair control system Proc. Fifth Int. ACM Conf. on Assistive Technologies (https://doi.org/10.1145/ 638249.638273)

[78] Kiguchi K and Hayashi Y 2012 An EMG-based control for an upper-limb power-assist exoskeleton robot IEEE Trans. Syst. Man Cybern. B 42 1064-71

[79] Rosen J, Brand M, Fuchs M B and Arcan M 2001 A myosignal-based powered exoskeleton system IEEE Trans. Syst. Man Cybernet A 31 210-22

[80] Rahmatillah A, Rahma O N, Amin M, Wicaksana S I, Ain K and Rulaningtyas R 2018 Post-stroke rehabilitation exosceleton movement control using EMG signal Int. J. Adv. Sci. Eng. Inf. Technol. 8 616-21

[81] Cheng K-T and Huang T-C 2009 What is flexible electronics? SIGDA Newsl. 39

[82] Crabb R and Treble F 1967 Thin silicon solar cells for large flexible arrays Nature 213 1223-4

[83] Garnier F et al 1990 An all-organic "soft" thin film transistor with very high carrier mobility $A d v$. Mater. 2 592-4

[84] Gustafsson G et al 1992 Flexible light-emitting diodes made from soluble conducting polymers Nature 357 477-9

[85] Garnier F, Hajlaoui R, Yassar A and Srivastava P 1994 All-polymer field-effect transistor realized by printing techniques Science 265 1684-6

[86] Someya T et al 2004 A large-area, flexible pressure sensor matrix with organic field-effect transistors for artificial skin applications Proc. Natl Acad. Sci. 101 9966-70

[87] Someya T et al 2005 Conformable, flexible, large-area networks of pressure and thermal sensors with organic transistor active matrixes Proc. Natl Acad. Sci. 102 12321-5

[88] Ma Z, Li S, Wang H, Cheng W, Li Y, Pan L and Shi Y 2019 Advanced electronic skin devices for healthcare applications J. Mater. Chem. B 7 173-97

[89] Sanderson K 2021 Electronic skin: from flexibility to a sense of touch Nature 591 685-7
[90] Yeo W-H et al 2013 Multi-functional electronics: multifunctional epidermal electronics printed directly onto the skin (Adv. Mater. 20/2013) Adv. Mater. 252772

[91] Kim D-H et al 2011 Epidermal electronics Science 333 838-43

[92] Huang X, Yeo W-H, Liu Y and Rogers J A 2012 Epidermal differential impedance sensor for conformal skin hydration monitoring Biointerphases 752

[93] Maiolo L, Polese D and Convertino A 2019 The rise of flexible electronics in neuroscience, from materials selection to in vitro and in vivo applications $A d v$. Phys. X 41664319

[94] Paradiso R and De Rossi D 2008 Advances in textile sensing and actuation for e-textile applications 30th Annual Int. Conf. IEEE Engineering in Medicine and Biology Society (IEEE) (https://doi.org/10.1109/IEMBS.2008.4649993)

[95] Hamedi M, Forchheimer R and Inganäs O 2007 Towards woven logic from organic electronic fibres Nat. Mater. $6357-62$

[96] Gorgutsa S, Bélanger-Garnier V, Ung B, Viens J, Gosselin B, LaRochelle S and Messaddeq Y 2014 Novel wireless-communicating textiles made from multi-material and minimally-invasive fibers Sensors 14 19260-74

[97] Lee Y-D and Chung W-Y 2009 Wireless sensor network based wearable smart shirt for ubiquitous health and activity monitoring Sens. Actuators B 140 390-5

[98] Kubicek J, Fiedorova K, Vilimek D, Cerny M, Penhaker M, Janura M and Rosicky J 2020 Recent trends, construction and applications of smart textiles and clothing for monitoring of health activity: a comprehensive multidisciplinary review IEEE Rev. Biomed. Eng. 1-1

[99] Acar G et al 2019 Wearable and flexible textile electrodes for biopotential signal monitoring: a review Electronics 8479

[100] Liu Z and Liu X 2015 Progress on fabric electrodes used in ECG signals monitoring J. Textile Sci. Technol. 1110

[101] Amitrano F et al 2020 Design and validation of an E-textile-based wearable sock for remote gait and postural assessment Sensors 206691

[102] Sung M, Jeong K and Cho G 2009 Establishing a measurement system for human motions using a textile-based motion sensor Int. Conf. on Human-Computer Interaction (Springer) (https://doi.org/ 10.1007/978-3-642-02580-8_86)

[103] Farina D et al 2010 High-density EMG E-textile systems for the control of active prostheses 2010 Annual Int. Conf. IEEE Engineering in Medicine and Biology (IEEE) (https://doi. org/10.1109/IEMBS.2010.5627455)

[104] Rus D and Tolley M T 2015 Design, fabrication and control of soft robots Nature 521 467-75

[105] Galloway K C, Becker K P, Phillips B, Kirby J, Licht S, Tchernov D, Wood R J and Gruber D F 2016 Soft robotic grippers for biological sampling on deep reefs Soft Robot. 3 23-33

[106] Bützer T, Lambercy O, Arata J and Gassert R 2021 Fully wearable actuated soft exoskeleton for grasping assistance in everyday activities Soft Robot. 8 128-43

[107] Shepherd R F et al 2011 Multigait soft robot Proc. Natl Acad. Sci. 108 20400-3

[108] Gifari M W et al 2019 A review on recent advances in soft surgical robots for endoscopic applications Int. J. Med. Robot. Comput. Assis. Surg. 15 e2010

[109] Peters B S et al 2018 Review of emerging surgical robotic technology Surg. Endosc. 32 1636-55

[110] Mekki M et al 2018 Robotic rehabilitation and spinal cord injury: a narrative review Neurotherapeutics 15 604-17

[111] Stoppa M and Chiolerio A 2014 Wearable electronics and smart textiles: a critical review Sensors 14 11957-92

[112] McLaren R et al 2016 A review of e-textiles in neurological rehabilitation: how close are we? J. Neuroeng. Rehabil. $131-13$

[113] Patel S et al 2012 A review of wearable sensors and systems with application in rehabilitation J. Neuroeng. Rehabil. 9 1-17 
[114] Lu N and Kim D-H 2014 Flexible and stretchable electronics paving the way for soft robotics Soft Robot. 1 53-62

[115] Yang Y et al 2020 Flexible actuators for soft robotics $A d v$. Intell. Syst. 21900077

[116] Panoulas K J, Hadjileontiadis L J and Panas S M 2010 Brain-computer interface (BCI): types, processing perspectives and applications Multimedia Services in Intelligent Environments (Berlin: Springer) pp 299-321

[117] Hochberg L R, Serruya M D, Friehs G M, Mukand J A, Saleh M, Caplan A H, Branner A, Chen D, Penn R D and Donoghue J P 2006 Neuronal ensemble control of prosthetic devices by a human with tetraplegia Nature 442 164-71

[118] Roland J L, Hacker C D, Breshears J D, Gaona C M, Hogan R E, Burton H, Corbetta M and Leuthardt E C 2013 Brain mapping in a patient with congenital blindness-a case for multimodal approaches Front. Hum. Neurosci. 7431

[119] Wolpaw J R and Boulay C B 2009 Brain signals for brain-computer interfaces Brain-computer Interfaces (Berlin: Springer) pp 29-46

[120] Abdulkader S N, Atia A and Mostafa M-S M 2015 Brain computer interfacing: applications and challenges Egyptian Inf. J. 16 213-30

[121] Blabe C H et al 2015 Assessment of brain-machine interfaces from the perspective of people with paralysis $J$. Neural. Eng. 12043002

[122] Collinger J L, Boninger M L, Bruns T M, Curley K, Wang W and Weber D J 2013 Functional priorities, assistive technology, and brain-computer interfaces after spinal cord injury J. Rehabil. Res. Dev. 50145

[123] McAdams E et al 1996 Factors affecting electrode-gel-skin interface impedance in electrical impedance tomography Med. Biol. Eng. Comput. 34 397-408

[124] Searle A and Kirkup L 2000 A direct comparison of wet, dry and insulating bioelectric recording electrodes Physiol. Meas. 21271

[125] Li G, Wang S and Duan Y Y 2017 Towards gel-free electrodes: a systematic study of electrode-skin impedance Sensors Actuators B 241 1244-55

[126] Salvo P et al 2012 A 3D printed dry electrode for ECG/EEG recording Sensors Actuators A 174 96-102

[127] Stauffer F et al 2018 Skin conformal polymer electrodes for clinical ECG and EEG recordings Adv. Healthcare Mater. 71700994

[128] Nakanishi M, Wang Y, Chen X, Wang Y-T, Gao X and Jung T-P 2017 Enhancing detection of SSVEPs for a high-speed brain speller using task-related component analysis IEEE Trans. Biomed. Eng. 65 104-12

[129] Kwak N-S, Müller K-R and Lee S-W 2017 A convolutional neural network for steady state visual evoked potential classification under ambulatory environment PLoS One 12 e 0172578

[130] Alvarez G D, Favaro F, Lecumberry F, Martin A, Oliver J P, Oreggioni J, Ramirez I, Seroussi G and Steinfeld L 2018 Wireless EEG system achieving high throughput and reduced energy consumption through lossless and near-lossless compression IEEE Trans. Biomed. Circuits Syst. 12 231-41

[131] Lin C-T, Chiu C-Y, Singh A K, King J-T, Ko L-W, Lu Y-C and Wang Y-K 2018 A wireless multifunctional SSVEP-based brain-computer interface assistive system IEEE Trans. Cogn. Dev. Syst. 11 375-83

[132] Mahmood M et al 2019 Fully portable and wireless universal brain-machine interfaces enabled by flexible scalp electronics and deep learning algorithm Nat. Mach. Intell. 1 412-22

[133] Xu J and Zhong B 2018 Review on portable EEG technology in educational research Comput. Human Behav. 81 340-9

[134] Shi M et al 2018 Towards portable SSVEP-based brain-computer interface using Emotiv EPOC and mobile phone 2018 Tenth Int. Conf. on Advanced Computational
Intelligence (ICACI) (IEEE) (https://doi.org/ 10.1109/ICACI.2018.8377615)

[135] Chen X, Zhao B, Wang Y, Xu S and Gao X 2018 Control of a 7-DOF robotic arm system with an SSVEP-based BCI Int. J. Neural Syst. 281850018

[136] Ren L et al 2018 Fabrication of flexible microneedle array electrodes for wearable bio-signal recording Sensors 181191

[137] Grozea C, Voinescu C D and Fazli S J 2011 Bristle-sensors-low-cost flexible passive dry EEG electrodes for neurofeedback and BCI applications $J$. Neural. Eng. 8025008

[138] Lepola P et al 2014 Screen-printed EEG electrode set for emergency use Sensors Actuators A 213 19-26

[139] Norton J J et al 2015 Soft, curved electrode systems capable of integration on the auricle as a persistent brain-computer interface Proc. Natl Acad. Sci. 112 3920-5

[140] Chan H-L et al 2018 Challenges and future perspectives on electroencephalogram-based biometrics in person recognition Front. Neuroinform. 1266

[141] Matiko J W et al 2015 Wearable EEG headband using printed electrodes and powered by energy harvesting for emotion monitoring in ambient assisted living Smart Mater. Struct. 24125028

[142] La T G et al 2018 Two-layered and stretchable e-textile patches for wearable healthcare electronics $A d v$. Healthcare Mater. 71801033

[143] Farina D and Negro F 2012 Accessing the neural drive to muscle and translation to neurorehabilitation technologies IEEE Rev. Biomed. Eng. 5 3-14

[144] Andersson E A, Nilsson J and Thorstensson A 1997 Intramuscular EMG from the hip flexor muscles during human locomotion Acta Physiol. Scand. 161 361-70

[145] Onishi H, Yagi R, Akasaka K, Momose K, Ihashi K and Handa Y 2000 Relationship between EMG signals and force in human vastus lateralis muscle using multiple bipolar wire electrodes J. Electromyogr. Kinesiol. 10 59-67

[146] Péter A et al 2019 Comparing surface and fine-wire electromyography activity of lower leg muscles at different walking speeds Front. Physiol. 101283

[147] Roy S H, De Luca C J and Schneider J 1986 Effects of electrode location on myoelectric conduction velocity and median frequency estimates J. Appl. Physiol. 61 1510-7

[148] Perry J, Easterday C S and Antonelli D J 1981 Surface versus intramuscular electrodes for electromyography of superficial and deep muscles Phys. Ther. 61 7-15

[149] Dimitrova N, Dimitrov G and Nikitin O 2002 Neither high-pass filtering nor mathematical differentiation of the EMG signals can considerably reduce cross-talk J. Electromyogr. Kinesiol. 12 235-46

[150] Lowery M M, Stoykov N S and Kuiken T A 2003 A simulation study to examine the use of cross-correlation as an estimate of surface EMG cross talk J. Appl. Physiol. 94 1324-34

[151] Merletti R, Rainoldi A and Farina D 2001 Surface electromyography for noninvasive characterization of muscle Exerc. Sport Sci. Rev. 29 20-25

[152] Pullman S L, Goodin D S, Marquinez A I, Tabbal S and Rubin M 2000 Clinical utility of surface EMG: report of the therapeutics and technology assessment subcommittee of the American Academy of Neurology Neurology 55 171-7

[153] Lee J W, Golgouneh A and Dunne L 2019 Comparative assessment of wearable surface EMG electrode configurations for biomechanical applications 49 th Int. Conf. on Environmental Systems

[154] De Marchis C et al 2016 Multi-contact functional electrical stimulation for hand opening: electrophysiologically driven identification of the optimal stimulation site J. Neuroeng. Rehabil. 13 1-9

[155] Duente T, Pfeiffer M and Rohs M 2016 On-skin technologies for muscle sensing and actuation Proc. 2016 ACM Int. Joint Conf. on Pervasive and Ubiquitous 
Computing: Adjunct (https://doi.org/

10.1145/2968219.2979136)

[156] Xu B et al 2016 An epidermal stimulation and sensing platform for sensorimotor prosthetic control, management of lower back exertion, and electrical muscle activation Adv. Mater. 28 4462-71

[157] Fall C et al 2018 Non-invasive and flexible electrodes based on multimaterial fiber for sEMG signal detection 2018 IEEE Life Sciences Conf. (LSC) (IEEE) (https://doi.org/ 10.1109/LSC.2018.8572212)

[158] Kim M K et al 2019 Flexible submental sensor patch with remote monitoring controls for management of oropharyngeal swallowing disorders Science Advances 5 eaay3210

[159] Rofes L, Vilardell N and Clavé P 2013 Post-stroke dysphagia: progress at last Neurogastroenterol. Motil. $25278-82$

[160] Wilkinson $\mathrm{N}$ et al 2019 A review of aerosol jet printing-a non-traditional hybrid process for micro-manufacturing Int. J. Adv. Manuf. Technol. 105 4599-619

[161] Khan M A et al 2021 EEG-based BCI systems for neurorehabilitation applications Brain and Behavior Computing (Boca Raton, FL: CRC Press) pp 189-219

[162] Horki P et al 2011 Combined motor imagery and SSVEP based BCI control of a 2 DoF artificial upper limb Med. Biol. Eng. Comput. 49 567-77

[163] Diez P F et al 2011 Asynchronous BCI control using high-frequency SSVEP J. NeuroEng. Rehabil. 8 1-9

[164] Sakurada T et al 2013 A BMI-based occupational therapy assist suit: asynchronous control by SSVEP Front. Neurosci. 7172

[165] Alasadi A, Claeyssens F and Allwood D 2018 Laser direct writing (LDW) of magnetic structures AIP Adv. 8056322

[166] Chen Z et al 2018 Rapid fabrication of microneedles using magnetorheological drawing lithography Acta Biomater. 65 283-9.

[167] Novaković Đ et al 2015 Screen printing Printing on Polymers: Fundamentals and Applications (Amsterdam: Elsevier) 247-62

[168] Heeravathi S and Christy A A 2020 Spin coating methods and applications-a review J. Xi'an Univ. Archit. Technol. 12 231-5

[169] Montazer M and Harifi T 2018 7-Nanocoating and lamination Nanofinishing of Textile Materials ed M Montazer and T Harifi (Cambridge: Woodhead Publishing) pp 95-107

[170] Vogel F, Träger F and Hubenthal F 2011 Noble metal nanoparticles: synthesis and optical properties J. Nanosci. Nanotechnol. 11 2368-75

[171] Barreca S, Wolf S L, Fasoli S and Bohannon R 2003 Treatment interventions for the paretic upper limb of stroke survivors: a critical review Neurorehabil. Neural Repair 17 220-6

[172] Alon G, Levitt A F and McCarthy P A 2007 Functional electrical stimulation enhancement of upper extremity functional recovery during stroke rehabilitation: a pilot study Neurorehabil. Neural Repair 21 207-15

[173] Hara Y 2008 Neurorehabilitation with new functional electrical stimulation for hemiparetic upper extremity in stroke patients J. Nippon Med. School 75 4-14

[174] Gharib N M et al 2015 Efficacy of electrical stimulation as an adjunct to repetitive task practice therapy on skilled hand performance in hemiparetic stroke patients: a randomized controlled trial Clin. Rehabil. 29 355-64

[175] Church C, Price C, Pandyan A D, Huntley S, Curless R and Rodgers H 2006 Randomized controlled trial to evaluate the effect of surface neuromuscular electrical stimulation to the shoulder after acute stroke Stroke 37 2995-3001

[176] Ferrante $S$ et al 2008 The effect of using variable frequency trains during functional electrical stimulation cycling Neuromodulation: Technol. Neural Interface 11 216-26
[177] Cauraugh J H and Kim S B 2003 Chronic stroke motor recovery: duration of active neuromuscular stimulation $J$ Neurol. Sci. 215 13-19

[178] Gobbo M et al 2014 Muscle motor point identification is essential for optimizing neuromuscular electrical stimulation use J. Neuroeng. Rehabil. 11 1-6

[179] Keller T and Kuhn A 2008 Electrodes for transcutaneous (surface) electrical stimulation J. Autom. Control 18 35-45

[180] Yang K et al 2018 Development of user-friendly wearable electronic textiles for healthcare applications Sensors 182410

[181] Yang K et al 2014 Screen printed fabric electrode array for wearable functional electrical stimulation Sens. Actuators A 213 108-15

[182] Malešević N M, Maneski L Z, Ilić V, Jorgovanović N, Bijelić G, Keller T and Popović D B 2012 A multi-pad electrode based functional electrical stimulation system for restoration of grasp J. Neuroeng. Rehabil. 966

[183] Loitz J C et al 2016 A flexible standalone system with integrated sensor feedback for multi-pad electrode FES of the hand Curr. Directions Biomed. Eng. 2 391-4

[184] Malešević N M et al 2010 Distributed low-frequency functional electrical stimulation delays muscle fatigue compared to conventional stimulation Muscle Nerve 42 556-62

[185] Sayenko D G et al 2014 Reducing muscle fatigue during transcutaneous neuromuscular electrical stimulation by spatially and sequentially distributing electrical stimulation sources Eur. J. Appl. Physiol. 114 793-804

[186] Nguyen R, Masani K, Micera S, Morari M and Popovic M R 2011 Spatially distributed sequential stimulation reduces fatigue in paralyzed triceps surae muscles: a case study Artif. Organs. 35 1174-80

[187] Veerbeek J M et al 2017 Effects of robot-assisted therapy for the upper limb after stroke: a systematic review and meta-analysis Neurorehabil. Neural Repair 31 107-21

[188] Hidler J, Nichols D, Pelliccio M and Brady K 2005 Advances in the understanding and treatment of stroke impairment using robotic devices Top. Stroke Rehabil. 12 22-35

[189] Kahn L E et al 2006 Robot-assisted reaching exercise promotes arm movement recovery in chronic hemiparetic stroke: a randomized controlled pilot study J. Neuroeng. Rehabil. 3 1-13

[190] Kahn L E, Lum P S, Rymer W Z and Reinkensmeyer D J 2014 Robot-assisted movement training for the stroke-impaired arm: does it matter what the robot does? J. Rehabil. Res. Dev. 43 619-30

[191] Gomez-Rodriguez M, Peters J, Hill J, Schölkopf B, Gharabaghi A and Grosse-Wentrup M 2011 Closing the sensorimotor loop: haptic feedback facilitates decoding of motor imagery J. Neural. Eng. 8036005

[192] Gomez-Rodriguez M et al 2011 Towards brain-robot interfaces in stroke rehabilitation 2011 IEEE Int. Conf. on Rehabilitation Robotics (IEEE) (https://doi.org/ 10.1109/ICORR.2011.5975385)

[193] Meyer T et al 2012 A brain-robot interface for studying motor learning after stroke 2012 IEEE/RSJ Int. Conf. on Intelligent Robots and Systems (IEEE) (https://doi.org/ 10.1109/IROS.2012.6385646)

[194] Brown E, Rodenberg N, Amend J, Mozeika A, Steltz E, Zakin M R, Lipson H, Jaeger H M 2010 Universal robotic gripper based on the jamming of granular material Proc. Natl Acad. Sci. 107 18809-14

[195] Galloway K et al 2015 Soft robotic grippers for biological sampling on deep reefs Soft Robot. 323-33

[196] Ilievski F et al 2011 Soft robotics for chemists Angew. Chem. 123 1930-5

[197] Marchese A, Onal C and Rus D 2014 Autonomous soft robotic fish capable of escape maneuvers using fluidic elastomer actuators Soft Robot. 1 75-87

[198] Bartlett N W, Tolley M T, Overvelde J T B, Weaver J C, Mosadegh B, Bertoldi K, Whitesides G M and Wood R J 
2015 A 3D-printed, functionally graded soft robot powered by combustion Science 349 161-5

[199] Chossat J-B, Park Y-L, Wood R J and Duchaine V 2013 A soft strain sensor based on ionic and metal liquids IEEE Sens. J. 13 3405-14

[200] Mengüç Y et al 2014 Wearable soft sensing suit for human gait measurement Int. J. Robot. Res. 33 1748-64

[201] Awad L N et al 2017 A soft robotic exosuit improves walking in patients after stroke Sci. Transl. Med. 99084

[202] Bae J et al 2018 Biomechanical mechanisms underlying exosuit-induced improvements in walking economy after stroke J. Exp. Biol. 221168815

[203] Di Natali C et al 2019 Design and evaluation of a soft assistive lower limb exoskeleton Robotica 37 2014-34

[204] Bae J et al 2018 A lightweight and efficient portable soft exosuit for paretic ankle assistance in walking after stroke 2018 IEEE Int. Conf. on Robotics and Automation (ICRA) (IEEE) (https://doi.org/10.1109/ICRA.2018.8461046)

[205] Awad L N et al 2020 Walking faster and farther with a soft robotic exosuit: implications for post-stroke gait assistance and rehabilitation IEEE Open J. Eng. Med. Biol. 1 108-15

[206] Stilli A et al 2018 AirExGlove-a novel pneumatic exoskeleton glove for adaptive hand rehabilitation in post-stroke patients 2018 IEEE Int. Conf. on Soft Robotics (Robosoft) (IEEE) pp 579-84

[207] Yap H K et al 2017 Design and preliminary feasibility study of a soft robotic glove for hand function assistance in stroke survivors Front. Neurosci. 11547

[208] Cheng N, Phua K S, Lai H S, Tam P K, Tang K Y, Cheng K K, Yeow R C-H, Ang K K, Guan C and Lim J H 2020 Brain-computer interface-based soft robotic glove rehabilitation for stroke IEEE Trans. Biomed. Eng. 67 3339-51

[209] Mcnulty J 2018 Game-changing skin-like electronics for stroke patients Health Manage. 18 232-4

[210] Stephens-Fripp B et al 2018 Reusable flexible concentric electrodes coated with a conductive graphene ink for electrotactile stimulation Front. Bioeng. Biotechnol. 6179

[211] Cömert A and Hyttinen J 2015 Investigating the possible effect of electrode support structure on motion artifact in wearable bioelectric signal monitoring Biomed. Eng. $141-18$

[212] Rotzler S, Krshiwoblozki M V and Schneider-Ramelow M 2021 Washability of e-textiles: current testing practices and the need for standardization Text. Res. J. 910040517521996727

[213] Ankhili A, Zaman S U, Tao X, Cochrane C, Končar V and Coulon D 2019 Washable embroidered textile electrodes for long-term electrocardiography monitoring Text. Leather Rev. 2 126-35

[214] Baribina N, Baltina I and Oks A 2018 Application of additional coating for conductive yarns protection against washing Key Engineering Materials vol 762 (Switzerland: Trans Tech Publ) 396-401

[215] Gaubert V et al 2020 Investigating the impact of washing cycles on silver-plated textile electrodes: a complete study Sensors 201739

[216] Komolafe A 2016 Reliability and interconnections for printed circuits on fabrics Doctoral Thesis Faculty of Physical Sciences and Engineering, University of Southampton

[217] Tao X, Koncar V, Huang T-H, Shen C-L, Ko Y-C and Jou G-T 2017 How to make reliable, washable, and wearable textronic devices Sensors 17673

[218] Tao X, Cochrane C and Koncar V 2020 Understanding the washing damage to textile ECG dry skin electrodes, embroidered and fabric-based; set up of equivalent laboratory tests Sensors 201272

[219] Björninen T et al 2014 Impact of recurrent washing on the performance of electro-textile UHF RFID tags 2014 IEEE RFID Technology and Applications Conf. (RFID-TA) (IEEE) (https://doi.org/10.1109/RFID-TA.2014.6934238)

[220] Giorgino T, Tormene P, Lorussi F, De Rossi D and Quaglini S 2009 Sensor evaluation for wearable strain gauges in neurological rehabilitation IEEE Trans. Neural. Syst. Rehabil. Eng. 17 409-15

[221] Hardy D A et al 2020 Wash testing of electronic yarn Materials 131228

[222] Veske P et al 2020 Development and washing reliability testing of a stretchable circuit on knit fabric Appl. Sci. 109057

[223] Yun S-S et al 2017 Exo-glove PM: an easily customizable modularized pneumatic assistive glove IEEE Robot. Autom. Lett. 2 1725-32

[224] Vatan H M F et al 2021 A review: a comprehensive review of soft and rigid wearable rehabilitation and assistive devices with a focus on the shoulder joint J. Intel. Robot. Syst. 102 1-24

[225] Kim D-H et al 2010 Dissolvable films of silk fibroin for ultrathin conformal bio-integrated electronics Nat. Mater. 9 511-7

[226] Viventi J et al 2011 Flexible, foldable, actively multiplexed, high-density electrode array for mapping brain activity in vivo Nat. Neurosci. 141599

[227] McFarland D J et al 2007 Toward Brain-computer Interfacing (Cambridge, MA: MIT Press)

[228] Lee K et al 2020 Mechano-acoustic sensing of physiological processes and body motions via a soft wireless device placed at the suprasternal notch Nat. Biomed. Eng. 4 148-58

[229] Kim S-W, Lee Y, Park J, Kim S, Chae H, Ko H and Kim J 2018 A triple-mode flexible E-skin sensor interface for multi-purpose wearable applications Sensors 1878

[230] Murphy M A et al 2019 An upper body garment with integrated sensors for people with neurological disorders-early development and evaluation BMC Biomed. Eng. 1 1-13 